\title{
État actuel des connaissances des procédés de bioréacteur à membrane pour le traitement et la réutilisation des eaux usées industrielles et urbaines \\ State-of-the-art review of the membrane bioreactor processes for urban and industrial wastewater treatment and reuse
}

\author{
Brahima Seyhi, Patrick Droguil, Géraldo Buelna, Jean-François Blais et Marc \\ Heran
}

Volume 24, numéro 3, 2011

Reçu le 22 janvier 2010, accepté le $1^{\mathrm{er}}$ décembre 2010

URI : https://id.erudit.org/iderudit/1006478ar

DOI : https://doi.org/10.7202/1006478ar

\section{Aller au sommaire du numéro}

\section{Éditeur(s)}

Université du Québec - INRS-Eau, Terre et Environnement (INRS-ETE)

ISSN

1718-8598 (numérique)

Découvrir la revue

Citer cet article

Seyhi, B., Droguil, P., Buelna, G., Blais, J.-F. \& Heran, M. (2011). État actuel des connaissances des procédés de bioréacteur à membrane pour le traitement et la réutilisation des eaux usées industrielles et urbaines. Revue des sciences de l'eau / Journal of Water Science, 24(3), 283-310.

https://doi.org/10.7202/1006478ar

\section{Résumé de l'article}

Les effluents issus des stations d'épuration des eaux usées industrielles et municipales contiennent des quantités non négligeables de polluants organiques, inorganiques et microbiens, qui sont rejetés dans l'environnement par voie directe, ou en suivant la filière de réutilisation (irrigation ou arrosage, etc.). Ces eaux résiduaires constituent l'une des principales sources de contamination des eaux de surface et souterraines (augmentation de la demande chimique en oxygène (DCO), coloration et eutrophisation des cours d'eau, etc.). Dans l'optique de palier le déficit croissant des ressources en eau destinées à la consommation humaine, ces eaux résiduaires sont de plus en plus soumises à des traitements poussés en vue d'une réutilisation. Cette réutilisation doit toujours être réalisée dans l'objectif de fournir une eau présentant, en continu, une qualité spécifique liée à l'usage attendu (eau de production, eau de lavage, eau de refroidissement, eau d'irrigation ou d'arrosage, etc.). Les procédés conventionnels peuvent s'avérer non adaptés, notamment par leur manque de fiabilité dans la qualité des eaux traitées et le risque encouru de contamination microbiologique. Pour faire face à cette importante problématique, les techniques membranaires, notamment les bioréacteurs à membrane (BRM), peuvent constituer une avenue potentielle de traitement et de réutilisation de ces effluents. L'intérêt de ces procédés réside dans leur aspect non polluant, leur facilité d'automatisation et leur capacité à éliminer simultanément les différents polluants en une seule étape de traitement. Ces technologies offrent la possibilité de clarifier et de désinfecter simultanément les eaux sans risque de formation de composés organo-halogénés. Dans cet article, les BRM sont situés par rapport aux techniques conventionnelles de traitement biologique d'effluents. Par la suite, un accent particulier est mis sur la présentation des connaissances actuelles concernant les principes de base des BRM, les critères d'application et les conditions d'opération qui influencent les performances de ces technologies. Les développements récents portant sur la modélisation mathématique de fonctionnement et de colmatage de ces modules sont également présentés. Finalement, les applications industrielles et les coûts d'implantation et d'opération de ces technologies sont brièvement discutés.
Ce document est protégé par la loi sur le droit d'auteur. L’utilisation des services d'Érudit (y compris la reproduction) est assujettie à sa politique d'utilisation que vous pouvez consulter en ligne.

https://apropos.erudit.org/fr/usagers/politique-dutilisation/ 


\section{ÉTAT ACTUEL DES CONNAISSANCES DES PROCÉDÉS DE BIORÉACTEUR À MEMBRANE POUR LE TRAITEMENT ET LA RÉUTILISATION DES EAUX USÉES INDUSTRIELLES ET URBAINES}

State-of-the-art review of the membrane bioreactor processes for urban and industrial wastewater treatment and reuse

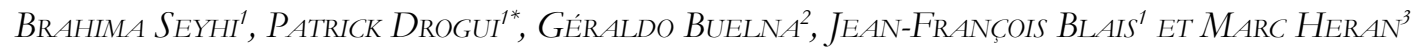

${ }^{1}$ Institut national de la recherche scientifique, Centre - Eau Terre Environnement, 490, rue de la Couronne, Québec (Québec) G1K 9A9, CANADA

${ }^{2}$ Centre de Recherche Industrielle du Québec (CRIQ), 333, rue Franquet, Québec (Québec) G1P 4C7, CANADA ${ }^{3}$ École Polytechnique Universitaire de Montpellier (EPUM), Université de Montpellier II, Sciences et Techniques du Languedoc, Place Eugène Bataillon, 34095, Montpellier Cedex 5, FRANCE

Reçu le 22 janvier 2010, accepté le $1^{\text {er }}$ décembre 2010

\section{RÉSUMÉ}

Les effluents issus des stations d'épuration des eaux usées industrielles et municipales contiennent des quantités non négligeables de polluants organiques, inorganiques et microbiens, qui sont rejetés dans l'environnement par voie directe, ou en suivant la filière de réutilisation (irrigation ou arrosage, etc.). Ces eaux résiduaires constituent l'une des principales sources de contamination des eaux de surface et souterraines (augmentation de la demande chimique en oxygène (DCO), coloration et eutrophisation des cours d'eau, etc.). Dans l'optique de palier le déficit croissant des ressources en eau destinéesà la consommation humaine, ceseaux résiduaires sont de plus en plus soumises à des traitements poussés en vue d'une réutilisation. Cette réutilisation doit toujours être réalisée dans l'objectif de fournir une eau présentant, en continu, une qualité spécifique liée à l'usage attendu (eau de production, eau de lavage, eau de refroidissement, eau d'irrigation ou d'arrosage, etc.). Les procédés conventionnels peuvent s'avérer non adaptés, notamment par leur manque de fiabilité dans la qualité des eaux traitées et le risque encouru de contamination microbiologique. Pour faire face à cette importante problématique, les techniques membranaires, notamment les bioréacteurs à membrane (BRM), peuvent constituer une avenue potentielle de traitement et de réutilisation de ces effluents. L'intérêt de ces procédés réside dans leur aspect non polluant, leur facilité d'automatisation et leur capacité à éliminer simultanément les différents polluants en une seule étape de traitement. Ces technologies offrent la possibilité de clarifier et de désinfecter simultanément les eaux sans risque de formation de composés organo-halogénés. Dans cet article, les BRM sont situés par rapport aux techniques conventionnelles de traitement biologique d'effluents. Par la suite, un accent particulier est mis sur la présentation des connaissances actuelles concernant les principes de base des BRM, les critères d'application et les conditions d'opération qui influencent les performances de ces technologies. Les développements récents portant sur la modélisation mathématique de fonctionnement et de colmatage de ces modules sont également présentés. Finalement, les applications

*Auteur pour correspondance:

Téléphone: 418-654-3119

T.élécopieur: 418-654-2600

Courriel : patrick.drogui@ete.inrs.ca 
industrielles et les coûts d'implantation et d'opération de ces technologies sont brièvement discutés.

Mots-clés : Bioréacteur à membrane, biotraitement, membrane immergée, colmatage, polluant organique réfractaire, désinfection, polluant inorganique, eaux usées.

\section{ABSTRACT}

Effluents from urban and industrial wastewater treatment plants contain organic (e.g., COD, BOD, total suspended solids, endocrine disrupting compounds), inorganic (e.g., phosphorus, ammonia nitrogen, nitrites and nitrates, metals) and microbial pollutants (e.g., bacteria, viruses, parasites), which are either directly discharged into the environment or reused for agricultural purposes. These wastewaters are often responsible for pollution of surface and groundwater (increasing the COD, colour and eutrophication of water, for example). In the context of finding solutions for water shortages, wastewaters are more and more frequently subjected to tertiary treatment for water reuse. The treatment of wastewater for reuse must yield water that meets specific quality criteria and is adapted to be reused as washing water, cooling water, process water, irrigation water or sprinkling water, among other uses. Conventional processes can be inappropriate, notably because of their inability to provide a consistently good quality of treated-water and because of the associated risk of microbial contamination. An alternate method can be the application of membrane bioreactors (MBR) for wastewater treatment and reuse. MBR are characterized by ease of operation, ease of automation, negligible equipment requirements for adding chemicals and their capacity to remove simultaneously organic, inorganic and microbial pollutants in the same reactor. This technology offers the possibility to simultaneously clarify and disinfect wastewaters without any risk of forming organochlorinated compounds. In this paper, MBR are first compared to conventional biological treatments, followed by a particular emphasis on the present state of knowledge about MBR, criteria of application and operating conditions that greatly influence the performance of these technologies. Recent developments in the modelling of the operating process and membrane fouling are also presented. Finally, industrial applications and operating and implementation costs are briefly discussed.

Key words: Membrane bioreactor, biotreatment, immersed membrane, fouling, refractory organic pollutant, disinfection, inorganic pollutant, wastewater.

\section{LISTE DES ABRÉVIATIONS}

$\Delta \mathrm{P}$

$\mu$

A

$\mathrm{AE}$

$\mathrm{a}_{\text {max }}$

APEO

$\mathrm{A}_{\mathrm{t}}$

BA

BHA

BPA

BRM

BRMe T

BRMi FC

BRMi P

CAPEX

$\mathrm{C}_{\mathrm{d}}$

$\mathrm{CF}$

COD

COT

CT

DBO

$\mathrm{DCO}$

$\mathrm{d}_{\mathrm{p}}$

$\mathrm{D}_{s}$

E1

E1-3G

E1-3S

E2

E2-3S

E2-G

EE2

$\mathrm{EH}$

EUD

EUI

EUM

EUS

F/M

FAA

$\mathrm{g}$

G

I

J $\mathrm{k}_{\mathrm{m}}$

$\mathrm{L}_{0}$

Perte de charge

Surface membranaire

Activité œestrogénique

Alkylphénol-polyéthoxylate

Surface totale des pores

Boue activée

Bactérie hétérotrophe aérobie

Bisphénol A

Bioréacteur à membrane membranes tubulaires membranes en fibres creuses membranes planes

d'investissement

de boue de diamètre $\mathrm{dp}$

Coliformes fécaux

Carbone organique dissous

Carbone organique total

Coliformes totaux fractale

Estrone

Estrone-3-glucuronide

Estrone-3-sulfate

$17 \beta$-œstradiol

Estradiol-3-sulfate

Estradiol-17 $\beta$-glucuronide

$17 \alpha$-éthinylestradiol

Équivalent-habitant

Eaux usées domestiques

Eaux usées industrielles

Eaux usées municipales

Eaux usées synthétiques organique

Formylalaminoantipyrine

Facteur de forme

Facteur géométrique

Intensité de cisaillement

Flux de perméat
Viscosité dynamique de la liqueur mixte

Limite surfacique maximale d'un pore

Bioréacteur à membrane à boucle externe avec

Bioréacteur à membrane immergée avec

Bioréacteur à membrane immergée avec

Capital expenditures ou dépenses

Coefficient de la force de levée d'une particule

Demande biochimique en oxygène

Demande chimique en oxygène

Diamètre des pores de la membrane

Dimension de la surface du pore selon la théorie

Rapport "Food/Microorganism» ou charge

Taux de croissance du colmatage

Coefficient qui reflète les effets de filtration

Constante caractérisant la longueur d'un pore 


\begin{tabular}{|c|c|}
\hline $\log \mathrm{K}_{\mathrm{ow}}$ & Coefficient de partition octanol/eau \\
\hline MBR & Membrane bioreactor \\
\hline $\mathrm{M}_{\mathrm{CD}}$ & $\begin{array}{l}\text { Masse de boue de la couche dynamique de } \\
\text { colmatage }\end{array}$ \\
\hline MES & Matières en suspension \\
\hline MF & Microfiltration \\
\hline MVES & Matières volatiles en suspension \\
\hline $\mathrm{N}_{\mathrm{T}}$ & Azote total \\
\hline NTK & Azote total Kjeldahl \\
\hline OPEX & $\begin{array}{l}\text { Operational expenditures ou dépenses de } \\
\text { fonctionnement, d'exploitation }\end{array}$ \\
\hline $\mathrm{P}_{\mathrm{a}}$ & Pression de l'alimentation \\
\hline $\mathrm{P}_{c}$ & Pression du concentrât \\
\hline PE & Perturbateurs endocriniens \\
\hline $\mathrm{p}_{\text {hydro }}$ & Pression hydrostatique \\
\hline PMS & Produits microbiens solubles \\
\hline $\mathrm{P}_{\mathrm{p}}$ & Pression du perméat \\
\hline $\mathrm{p}_{\text {pomp }}$ & Pression de succion \\
\hline $\mathrm{P}_{\mathrm{T}}$ & Phosphore total \\
\hline PTM & Pression transmembranaire \\
\hline PTFE & Polytétrafluoroéthylène \\
\hline $\mathrm{PP}$ & Polypropylène \\
\hline PES & Polyéthersulfone \\
\hline PS & Polysulfone \\
\hline PAN & Polyacrylonitrilique \\
\hline Q & Débit à travers la membrane \\
\hline $\mathrm{R}_{\mathrm{C}}$ & Résistance de colmatage \\
\hline $\mathrm{R}_{\mathrm{CD}}$ & Résistance de formation de couche dynamique \\
\hline $\mathrm{R}_{\mathrm{G}}$ & Résistance du gâteau \\
\hline $\mathrm{R}_{\mathrm{irr}}$ & Résistance irréversible \\
\hline $\mathrm{R}_{\mathrm{m}}$ & Résistance de la membrane \\
\hline $\mathrm{R}_{\mathrm{rev}}$ & Résistance réversible \\
\hline $\mathrm{R}_{\mathrm{T}}$ & Résistance totale de la membrane \\
\hline$S$ & Concentration de substrat \\
\hline$S_{C}$ & Seuil de coupure de la membrane \\
\hline SPE & Substances polymériques extracellulaires \\
\hline STEP & Station d'épuration des eaux usées \\
\hline $\mathrm{T}$ & Température \\
\hline $\mathrm{t}_{\mathrm{f}}$ & Temps de filtration \\
\hline TRH & Temps de rétention hydraulique \\
\hline TRS & Temps de rétention de solides \\
\hline UF & Ultrafiltration \\
\hline $\mathrm{V}_{\mathrm{p}}$ & Volume de perméat \\
\hline $\mathrm{v}_{\mathrm{T}}$ & Vitesse d'écoulement transversal \\
\hline $\mathrm{X}_{\mathrm{b}}$ & $\begin{array}{l}\text { Concentration de biomasse ou de MES dans le } \\
\text { bioréacteur }\end{array}$ \\
\hline$Y_{b}$ & Coefficient de compression de la boue \\
\hline$\alpha$ & Résistance spécifique \\
\hline$\beta$ & $\begin{array}{l}\text { Coefficient du taux d'érosion de la biomasse } \\
\text { dynamique }\end{array}$ \\
\hline$\Delta_{\text {pax }}$ & Chute de pression du flux de perméat \\
\hline$\theta$ & Âge des boues \\
\hline
\end{tabular}

\section{INTRODUCTION}

Les eaux usées industrielles (EUI), municipales (EUM) et domestiques (EUD) contiennent des quantités non négligeables de polluants organiques, inorganiques et microbiens. Ces eaux résiduaires constituent l'une des principales sources de contamination des eaux de surface et souterraines. Des stratégies de traitement efficaces devront être adoptées par les industries, les scientifiques et les gestionnaires pour atteindre, de manière optimale, les normes de rejets qui évoluent vers une sévérité croissante, aussi bien en ce qui concerne les concentrations en polluants que les débits. Par exemple, pour les rejets en milieu naturel des EUD, il a ainsi été défini des zones dites normales, pour lesquelles le traitement est principalement axé sur l'élimination des fractions particulaires et des pollutions carbonées, et des zones dites sensibles, pour lesquelles une élimination complémentaire des fractions azotées, phosphatées et microbiennes est nécessaire. Pour les EUD, les procédés dits conventionnels, qu'ils soient intensifs (ex. : boues activées, lits bactériens, biofiltres aérobies), extensifs (ex. : lagunage, système d'infiltration) ou combinés, peuvent répondre aux exigences de rejets en présentant chacun des performances plus ou moins fiables, du fait de leur sensibilité à des variations brusques de charges à traiter (cas des systèmes à cultures libres), de l'état de floculation des populations épuratives (cas des boues activées) ou de défauts de maitrise de la répartition de la biomasse et des écoulements au sein de garnissages poreux (systèmes à cultures fixées dans des lits à ruissellement, voire biofiltres). Pour les EUI, des règlements précisent également les conditions de rejet en milieu naturel ou dans le réseau d'égout municipal. Cependant, on observe un intérêt croissant pour des systèmes permettant une réutilisation partielle ou totale des eaux traitées. Cette réutilisation des eaux traitées est intéressante pour plusieurs raisons : i) elle permet de palier le déficit croissant des eaux destinées à la consommation humaine; ii) elle permet de préserver les milieux naturels en minimisant les besoins en eau, quels qu'en soient l'usage et les rejets; et iii) elle peut également se justifier économiquement par rapport au traitement d'une eau de surface ou souterraine de qualité dégradée qui oblige à faire appel à des systèmes de traitement de plus en plus complexes.

Pour faire face à cette importante problématique, les techniques membranaires, notamment les BRM, peuvent constituer une avenue potentielle de traitement et de réutilisation de ces effluents. L'intérêt de ces procédés réside dans leur aspect non polluant, leur facilité d'automatisation et leur capacité à éliminer simultanément les polluants de types organique, inorganique et microbien en une seule étape de traitement. Ces technologies offrent la possibilité de clarifier et de désinfecter simultanément les eaux sans risque de formation de composés organo-halogénés. 


\section{MISE EN CONTEXTE DE}

\section{L'UTILISATION DU BIORÉACTEUR À MEMBRANE}

Les EUM et EUI contiennent une panoplie de polluants de types polluants organiques (DCO, $\mathrm{DBO}_{5}, \mathrm{MES}$, perturbateurs endocriniens, etc.), inorganiques (phosphore, azote ammoniacal, nitrites et nitrates, métaux, etc.) et microbiens (bactéries, virus, parasites, etc.) qui échappent à l'épuration classique des eaux usées. Il existe plusieurs systèmes d'épuration des eaux usées (les procédés biologiques, chimiques, électrochimiques, physiques, etc.) et leur choix nécessite la prise en considération de plusieurs facteurs tels que la composition des eaux usées, la sensibilité du milieu récepteur, les normes de rejets et les objectifs de réutilisation des eaux traitées, etc. Les procédés d'épuration par voie biologique (boues activées, lits bactériens, biofiltres, lagunage, etc.) sont souvent utilisés dans le traitement des EUD, EUM et EUI. En effet, ces procédés, où les bactéries sont les principaux acteurs de la dégradation des polluants, sont relativement peu coûteux et faciles à mettre en place. Parmi ces procédés biologiques, le système de traitement par boues activées (BA) est le plus utilisé. Il constitue l'un des traitements les plus adéquats pour traiter, à grande échelle, des effluents chargés de composés biodégradables.

L'unité de BA se compose généralement d'un bassin d'aération où s'effectuent les processus de transfert et de métabolisation, et d'un clarificateur où s'effectue la séparation des solides (biomasses) et de l'eau épurée. Cette étape de séparation joue deux rôles essentiels : i) minimiser la teneur en MES dans l'eau traitée; et ii) retenir la biomasse épuratoire pour la renvoyer vers le bioréacteur afin d'y maintenir une concentration en biomasse adaptée à l'épuration attendue. La fiabilité de cette étape est donc déterminante pour la qualité de l'eau traitée, mais aussi pour la maîtrise des processus biologiques en empêchant le « lessivage » du réacteur biologique (diminution progressive de la concentration en biomasse, et donc de l'activité dans le réacteur, en raison d'une vitesse de croissance inférieure des espèces épuratives au flux spécifique d'extraction du bioréacteur). Il est ainsi primordial de maîtriser la bonne décantabilité des boues, ce qui, malheureusement, peut échapper ponctuellement aux opérateurs du fait de la variabilité de la composition de l'intrant ou de l'apparition de conditions de réaction non optimales (introduction accidentelle de toxiques, baisse de température, teneur en oxygène dissous insuffisante, écart de $\mathrm{pH}$, concentration en biomasse excessive, etc.), entraînant une défloculation ou l'apparition de flocs à faible décantabilité (foisonnement des boues). Il n'est donc pas rare d'observer, sur de tels systèmes, des dysfonctionnements préjudiciables pour l'environnement ou pour un objectif de réutilisation.
Pour y remédier, l'étape de décantation devra être améliorée en utilisant une barrière infranchissable par les espèces épuratoires. Le choix de cette barrière s'est ainsi porté sur l'utilisation de membranes poreuses (MF ou UF). Cette association d'un système biologique et de l'étape de séparation sur membranes poreuses a donné naissance au procédé appelé «bioréacteur à membranes (BRM)".

\section{PRÉSENTATION DU BRM}

Le BRM est une technologie hybride qui couple un système biologique et une étape de séparation sur membrane poreuse. Cette technologie est une amélioration du système biologique à boues activées, vieille de près de 100 ans, qui se caractérise par le remplacement du traditionnel décanteur secondaire par une unité de filtration membranaire (XING et al., 2000), dont la sélectivité remarquable représente une barrière infranchissable par les espèces épuratoires, quel que soit leur état de floculation. Le processus de métabolisation avec ses deux aspects (anabolisme et catabolisme) observés dans un système de boues activées est similaire à celui mis en jeu dans un BRM. La technologie de BRM a été introduite pour la première fois dans les années 1960, mais l'intérêt grandissant de son application au traitement des eaux usées est réellement apparue quelques années plus tard. Les premières installations commercialisées ont vu le jour dans les années 1970 et 1980 pour des marchés atypiques (effluents de croisière navale, lixiviats de décharge et effluents industriels très concentrés) (STEPHENSON et al., 2000). Puis, en Europe par exemple, c'est dans les années 1990 que les premiers systèmes de BRM ont été mis en opération pour le traitement des EUM (LE-CLECH et al., 2006; LESJEAN et HUISJES, 2008; MELIN et al., 2006). Le développement a fait apparaître deux types de configurations : le BRM à boucle externe et le BRM immergé (Figures 1a et 1b).

\subsection{Bioréacteur à membrane à boucle externe}

Le BRM à boucle externe (Figure 1a) constitue la première génération (LESJEAN et HUISJES, 2008). Il se caractérise par un module membranaire en positionnement externe par rapport au bioréacteur de type boues activées. Le contenu (liqueur mixte) de ce dernier est injecté sur la membrane et la biomasse retenue par filtration membranaire est recyclée afin de maintenir une concentration beaucoup plus élevée dans le bioréacteur. Le BRM à boucle externe est mieux adapté aux eaux usées caractérisées par des températures relativement élevées (ex. : $40{ }^{\circ} \mathrm{C}$ ), des concentrations élevées (ex. : $10500 \mathrm{mg} \mathrm{DCO} \bullet \mathrm{L}^{-1}$ ) et des $\mathrm{pH}$ élevés (YANG et al., 2006). Il est peu compatible avec le traitement de débits d'eaux importants et peu concentrés comme le sont les EUM. Le BRM 


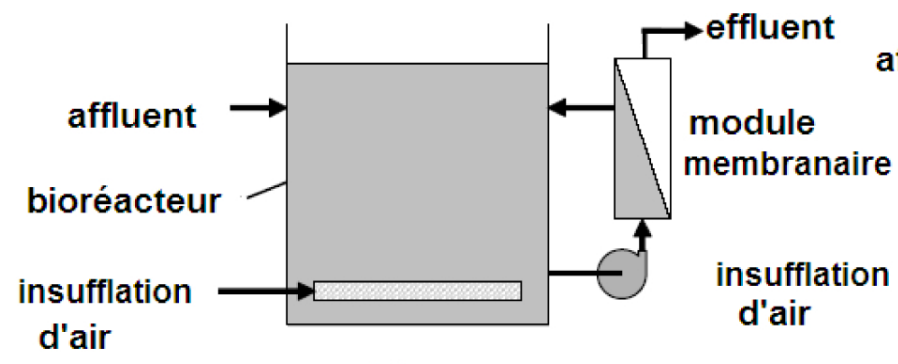

(a)

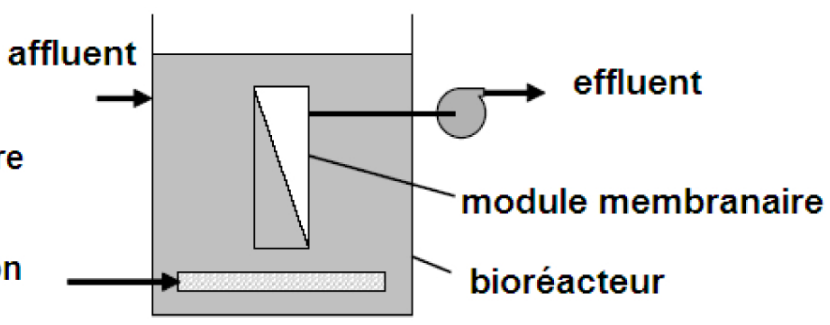

(b)

Figure 1. Configurations des BRM : a) BRM à boucle externe, b) BRM immergé (adapté de NG et KIM, 2007). BRM configurations: a) external loop BRM, b) submerged BRM (adapted from NG and KIM, 2007).

à boucle externe a longuement été appliqué aux petites entités telles que les eaux usées de navires, les lixiviats de décharge ou les effluents industriels (YANG et al., 2006). Dans cette configuration, le maintien de la perméabilité membranaire à un niveau économiquement rentable est obtenu en pratiquant une filtration dite tangentielle, imposant une circulation de la suspension dans le module membranaire à grandes vitesses $(0,5$ à $\left.4 \mathrm{~m} \bullet \mathrm{s}^{-1}\right)$, éventuellement combinée à une circulation gazeuse (VERA et al., 2000). La littérature fait également mention de l'application BRM à boucle externe pour le traitement des eaux souterraines en vue de la production d'eau potable (LESJAN et HUISJES, 2008). Cependant, la demande énergétique élevée fait en sorte qu'aucune croissance significative du marché des BRM à boucle externe n'est observée, comparativement à celle des BRM immergés (LESJAN et HUISJES, 2008).

\subsection{Bioréacteur à membrane immergée}

Dans l'optique de diminuer les coûts de fonctionnement dus à cette circulation intense de la suspension dans les modules externes, une seconde génération a été développée au début des années 1990. Cette nouvelle configuration repose sur l'immersion des membranes ou des modules (en général des membranes planes ou en fibres creuses) directement dans les boues activées (Figure 1b). La filtration (pseudo-frontale) est accomplie par l'application d'une pression négative du côté du perméat et par injection d'air directement sous et à l'intérieur du module membranaire, afin de maintenir les particules en suspension et nettoyer la surface externe de la membrane. Cette configuration présente des avantages sur le plan technico-économique où l'injection d'air est moins coûteuse qu'une circulation de la suspension sous condition turbulente pour les membranes à boucle externe. Le tableau 1 répertorie une liste non exhaustive de quelques problèmes observés par les opérateurs lors du fonctionnement des BRM à grande échelle (BRM à boucle externe versus BRM immergé). L'apparition des BRM immergés a ouvert le marché municipal $\mathrm{du}$ traitement des eaux par des techniques membranaires (LESJEAN et HUISJES, 2008). En effet, le BRM immergé est largement utilisé pour traiter les EUD et EUM (YANG et al., 2006). En 2005, en Europe, les membranes immergées représentaient $97 \%$ des surfaces membranaires totales installées et plus de $99 \%$ des surfaces membranaires totales annuelles de 2003 à 2005 (LESJEAN et HUISJES, 2008). Les faibles flux sous lesquels opère le BRM immergé $\left(15\right.$ à $50 \mathrm{~L} \cdot \mathrm{h}^{-1} \bullet \mathrm{m}^{-2}$ comparativement à 50 à $120 \mathrm{~L} \cdot \mathrm{h}^{-1} \bullet \mathrm{m}^{-2}$ pour les membranes à boucle externe) impliquent la nécessité d'avoir une surface membranaire élevée, et donc l'utilisation simultanée de plusieurs membranes (GANDER et al., 2000; MARROT et al., 2004). Les membranes immergées nécessitent donc un capital d'installation plus élevé, mais elles apportent une réduction significative des coûts d'exploitation en comparaison avec le BRM à boucle externe. Cependant, tandis que le bioréacteur requiert un bon transfert d'oxygène (nécessité de fines bulles d'oxygène), la membrane requiert des bulles de dimension plus grande, afin de maintenir une bonne perméabilité et de réduire son colmatage. Pour des raisons de maintenance, les membranes sont, pour la plupart des fabricants, maintenant immergées dans un bassin dédié à la filtration, ce qui oblige des recirculations importantes (300 à $400 \%$ du débit du perméat) afin de limiter l'augmentation de la concentration dans le bassin membranaire. La présence du bassin de filtration augmente les coûts d'investissement (construction du bassin de filtration) et de fonctionnement (pompe de recirculation, aération supplémentaire de la membrane dans le bassin de filtration) et pourrait remettre en cause l'un des facteurs clés en faveur du choix du BRM à l'échelle municipale. Le BRM immergé avec un bassin de filtration (configuration de type "outside") est préféré, surtout pour les sites avec des pics de charges élevés et des exigences plus strictes en matière de rejets des nutriments (BREPOLS et al., 2005; LESJEAN et al., 2008). Un effluent de concentration en azote $<2,2 \mathrm{mg} \cdot \mathrm{L}^{-1} \mathrm{~N}$ (norme de rejets en Hollande) peut être atteint à la sortie d'une STEP équipée d'un BRM immergé en configuration "outside", compte tenu du fait que la dénitrification peut y être bien contrôlée (AMEDEUS, 2008). Le BRM immergé en configuration de type "outside" fournit également des conditions opératoires plus flexibles et permet une optimisation indépendante des processus biologique et membranaire. Une étude comparative récente, effectuée en Europe sur deux STEP, a indiqué de très faibles différences entre les deux configurations de 
Tableau 1. Problèmes technologiques communs des BRM (YANG et al., 2006).

Table 1. Common technology problems with MBR (YANG et al., 2006).

\begin{tabular}{ll}
\hline BRM & Problèmes \\
\hline \multirow{3}{*}{$\begin{array}{l}\text { BRM immergé et BRM à } \\
\text { boucle externe }\end{array}$} & $\begin{array}{l}\text { Apparition de mousse dans le bioréacteur } \\
\text { Colmatage des membranes } \\
\text { Faible transfert d'oxygène }\end{array}$ \\
& Impacts des solides en suspension issus des effluents bruts \\
BRM à boucle externe & $\begin{array}{l}\text { Impact de la température du BRM sur ses performances } \\
\text { Impact de l'air entraîné sur le fonctionnement de la pompe de succion } \\
\text { Nettoyage membranaire très rigoureux } \\
\text { Colmatage de la membrane pendant le backpulsing du perméat } \\
\text { FRM immergé }\end{array}$ \\
& $\begin{array}{l}\text { Coible perméabilité membranaire contrairement à la perméabilité espérée } \\
\text { bioréacteur }\end{array}$ \\
\hline
\end{tabular}

BRM à membrane immergée (configurations de types "inside" et "outside"), en matière de consommation énergétique (VAN BENTEM et al., 2007). Dans la configuration de type "inside", la membrane est directement immergée dans la suspension à traiter. En effet, VAN BENTEM et al. (2007) ont rapporté que les STEP de Nordkanal en Allemagne (BRM immergé en configuration "inside") et de Varsseveld en Hollande (BRM immergé en configuration "outside") avaient des consommations énergétiques sensiblement identiques. La consommation énergétique de la STEP de Nordkanal était de $0,80 \mathrm{kWh} \cdot \mathrm{m}^{-3}$, tandis que celle de Varsseveld était de $0,88 \mathrm{kWh} \cdot \mathrm{m}^{3}$ (VAN BENTEM et al., 2007). Cependant, TAO et al. (2005) ont observé un ratio air/perméat plus élevé pour des BRM pilotes immergés opérés en configuration "outside", comparativement au BRM immergé fonctionnant en configuration "inside" (Tableau 2). Ces auteurs ont également observé une réduction de $8 \%$ des coûts énergétiques dans la configuration "inside".

\subsection{Structure et composition des membranes}

Dans l'optique de retenir totalement des espèces épuratoires et divers types de polluants (microbiens, organiques et inorganiques) présents dans le réacteur ou dans l'eau à traiter, le seuil de coupure des membranes est souvent choisi dans le domaine de la microfiltration (diamètre moyen du diamètre des pores : 0,05 à $0,4 \mu \mathrm{m}$ ) ou dans le domaine de l'ultrafiltration (10 à $50 \mathrm{~nm}$ ) pour assurer, notamment, la rétention des virus (GRASMICK etal., 2009). Ces membranes peuvent être de type organique ou inorganique. Les membranes organiques sont, en général, fabriquées de matériau polymérique hydrophobe (polytétrafluoroéthylène (PTFE), polypropylène (PP), etc.) ou hydrophile (polyéthersulfone (PES), polysulfone (PS), polyacrilonitrilique (PAN), etc.) (BERLAND et JUERY, 2002; CARDOT, 1999). Ces membranes sont souvent constituées de fibres creuses sous forme de minces tubes poreux. Les fibres poreuses sont soit regroupées dans un cylindre, le tout constituant un module tubulaire, soit regroupées en faisceau (module en fibres creuses). Dans le module tubulaire, l'eau à traiter entre à un bout des tubes et l'excès d'eau polluée sort à l'autre bout, tandis que l'eau filtrée est récupérée sur les côtés. La partie active de la membrane se trouve à l'intérieur de ces tubes et l'eau à traiter circule à l'intérieur. On distingue deux types de configurations possibles des modules en fibres creuses (BOUCHARD et al., 2000; BUISSON et al., 1998). Dans un premier cas, l'eau circule à l'intérieur des fibres et le perméat est récupéré à l'extérieur des fibres (configuration Int.-Ext.). Dans le deuxième cas, l'eau circule à l'extérieur des fibres et le perméat est récupéré à l'intérieur des fibres (configuration Ext.-Int.). Les membranes organiques peuvent également être soit empilées en mille-feuilles séparés par des cadres intermédiaires qui assurent la circulation de l'eau (modules plans), soit enroulées sur elles-mêmes autour d'un tube poreux (modules spiralés) (BERLAND et JUERY, 2002).

Les membranes inorganiques sont préparées à partir de polymère inorganique (alumine, zircone, oxyde de titan, carbone, acier) et sont constituées, tout comme les membranes organiques, d'une fine couche active efficace supportée par une paroi plus épaisse, permettant de donner à l'ensemble une plus grande résistance à la pression. Ces membranes minérales sont des blocs macroporeux, souvent cylindriques, percés de canaux autour desquels se situe la partie active de la membrane (MOULIN, 1990). L'eau circule dans les tubes et une partie traverse la couche active, puis se diffuse dans la partie poreuse et ressort à la périphérie du bloc. Parmi ces membranes (organiques ou inorganiques), on distingue des membranes à structures symétrique, asymétrique et composite (Figures 2a, 2b et 2c) (BERLAND et JUERY, 2002; CARDOT, 1999). Les membranes à structure asymétrique sont constituées d'une fine pellicule appelée " peau ", déposée sur un support poreux plus 
Tableau 2. Comparaison des consommations énergétiques $\left(\mathrm{kWh} \cdot \mathrm{m}^{-3}\right)$ de trois BRM pilotes opérés en parallèle sous différentes conditions (TAO et al., 2005).

Table 2. Comparison of energy consumption ( $\left(\mathrm{Wh}_{\left.\mathrm{m}-{ }^{3}\right)}\right.$ for three MBR pilots operated in parallel under different conditions (TAO et al., 2005).

\begin{tabular}{lcc}
\hline & Flux d'air $\left(\mathrm{m}^{3} \bullet \mathrm{m}^{-2} \bullet \mathrm{h}^{-1}\right)$ & Consommation d'énergie $\left(\mathrm{kWh} \bullet \mathrm{m}^{-3}\right)$ \\
\hline BRM A (Outside) & 1,2 & 1,0 \\
BRM B (Inside) & 1,0 & 0,8 \\
BRM C (Outside) & 1,3 & 1,1 \\
\hline
\end{tabular}

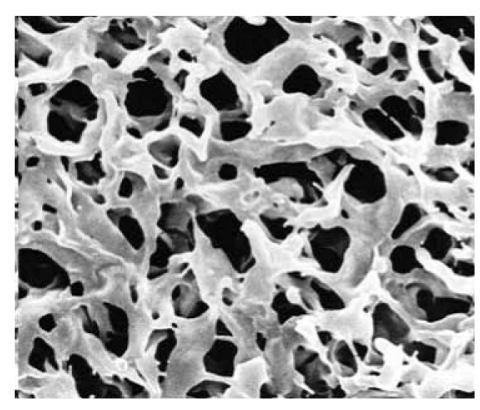

(a)

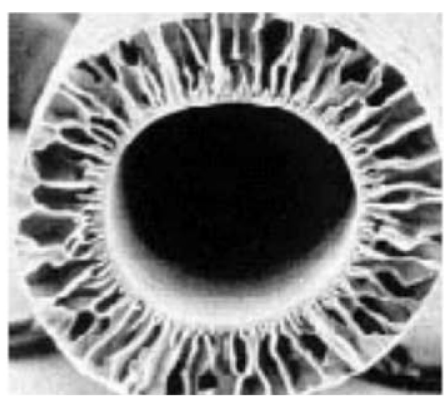

(b)

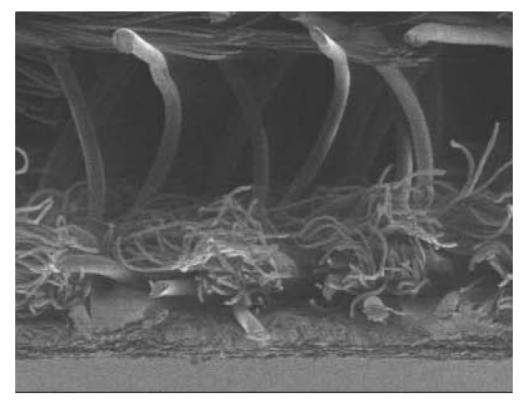

(c)

Figure 2. Structures de membranes : a) structure symétrique, b) structure asymétrique, c) structure composite. Structures of membranes: a) symmetrical structure, b) asymmetrical structure, c) composite structure.

grossier de même nature chimique, et dont le rôle est d'assurer à l'ensemble de bonnes propriétés mécaniques. Les membranes à structure composite se différencient des précédentes par le fait qu'elles sont obtenues en déposant la " peau " sélective sur un support préexistant de nature chimique différente. Les membranes à structure symétrique possèdent une distribution régulière et uniforme des pores sur toute leur épaisseur (utilisées seulement en microfiltration).

\subsection{Mise en ouvre et paramètres opératoires}

La mise en œuvre d'un BRM nécessite la prise en compte d'un certain nombre de facteurs qui gouvernent chaque opération de base, notamment le dimensionnement du BRM, les conditions hydrodynamiques de fonctionnement, lastructure de la membrane, la configuration du module membranaire, le coût de la membrane, etc. Le temps de rétention hydraulique (TRH) va déterminer le temps dont on dispose pour dégrader les composés solubles, et donc le volume du bioréacteur. Le temps de rétention solide (TRS, ou âge des boues : $\theta$ ) correspond au temps requis pour dégrader les composés particulaires et permettre le renouvellement/lyse de la biomasse épuratoire. Il influencera donc directement la teneur en MES dans le bassin. Ensuite, pour un bon fonctionnement biologique, il faudra s'assurer de ne pas être en oxygène limitant. Outre les facteurs précités, le choix des caractéristiques du système de filtration doit aussi intégrer la nature spécifique des suspensions présentes dans le bioréacteur à membrane, et notamment, leur concentration importante en biomasse cellulaire (8 à $\left.25 \mathrm{~g} \bullet \mathrm{L}^{-1}\right)$, mais également leur caractère évolutif en fonction des contraintes hydrodynamiques et biologiques imposées. Une parfaite connaissance des relations entre les paramètres opératoires et paramètres biologiques (temps de rétention hydraulique, âge des boues, pression transmembranaire, flux critique, rendement, activité de la biomasse, concentration de biomasse, etc.), ainsi que les paramètres physiques (filtration, durabilité, colmatage des membranes, rétro-lavage, etc.) est nécessaire pour optimiser le design et le fonctionnement du BRM. La mise en œuvre des processus biologiques impliqués dans la biodégradation des composés récalcitrants nécessite une acclimatation de la biomasse cellulaire à la dégradation de la pollution. Un des enjeux majeurs de la mise en œuvre industrielle d'un BRM est de maintenir, en cours d'opération, la perméabilité membranaire à des valeurs économiquement acceptables, et donc de minimiser le colmatage. En général, les paramètres suivis en cours d'opération sont la pression transmembranaire (PTM), la vitesse d'écoulement du flux d'eau $\left(\mathrm{v}_{\mathrm{T}}\right)$, les concentrations de MES dans l'eau à traiter et dans le bioréacteur, le temps de rétention hydraulique (TRH), la concentration d'oxygène dissous, la consommation spécifique d'oxygène, etc.

\subsubsection{Pression transmembranaire (PTM)}

La pression transmembranaire est la pression de filtration membranaire s'exerçant de part et d'autre de la membrane. 
Dans le BRM à boucle externe, la PTM est obtenue par l'équation 1 (CARDOT, 1999) :

$$
\mathrm{PTM}=\frac{\mathrm{P}_{\mathrm{a}}+\mathrm{P}_{\mathrm{c}}}{2}-\mathrm{P}_{\mathrm{p}}
$$

Dans cette équation, « $\mathrm{P}$ a, $\mathrm{Pa}$ » est la pression d'alimentation, « $\mathrm{P}, \mathrm{Pa}$ » est la pression du perméat et « $\mathrm{P}, \mathrm{Pa}$ » est la pression du concentrât. Les opérations de filtration peuvent être conduites en imposant soit un gradient de pression, soit un flux de perméation. Le BRM doit toujours être opéré en dessous d'une certaine PTM appelée pression critique. La valeur de la pression critique dépend de la nature chimique de la membrane et est souvent donnée par le fournisseur.

La plupart des installations fonctionne à débit constant et une pompe d'aspiration est utilisée pour les membranes immergées, même si des pressions transmembranaires de quelques dixièmes de bars sont déjà assurées par les hauteurs d'eau présente dans les bassins, ou par le niveau de rejet du perméat. Le flux est un paramètre décisif dans l'évolution de la PTM. Même si la filtration est effectuée en dessous des conditions critiques, un changement de la PTM est observé. Selon ORANTES et al. (2006), dans un BRM opéré à un flux $\mathrm{J}<5 \mathrm{~L} \bullet \mathrm{h}^{-1} \bullet \mathrm{m}^{-2}$ avec une membrane de type polysulfone (diamètre de pore $=0,1 \mu \mathrm{m}$ ), la perméabilité évolue suivant deux paliers (Tableau 3) : i) entre 0 et $500 \mathrm{~h}$ environ, la perméabilité change très lentement; et ii) au delà de $550 \mathrm{~h}$ de filtration, une diminution brusque de la perméabilité apparaît, caractérisée par une augmentation de la PTM, qui nécessite une régénération de la membrane.
3.4.2 Temps de rétention hydraulique (TRH), temps de rétention de solides (TRS ou âge des boues: $\theta$ ) et MES

Le TRH et le TRS (l'âge des boues : $\theta$ ) sont des paramètres importants dans les processus biologiques. La présence de la membrane permet au BRM de fonctionner à de fortes concentrations de biomasse et des âges des boues élevés, indépendamment du TRH. Le TRS varie généralement entre 25 et 80 jours et la concentration de biomasse est comprise entre 8 et $25 \mathrm{~g} \bullet \mathrm{L}^{-1}$ (CIRJA et al., 2008). Comparativement au procédé conventionnel de boues activées, la possibilité de travailler avec de fortes concentrations en biomasse et des $\theta$ élevés va présenter des atouts particuliers pour intensifier les performances de traitement. Pour une même charge volumique à traiter, à vitesse spécifique d'épuration équivalente, les vitesses apparentes de réaction sont intensifiées et la taille $\mathrm{du}$ volume réactionnel est d'autant plus réduite que la concentration en biomasse est augmentée (GRASMICK et al., 2009; XING et al., 2000). Les $\theta$ élevés imposés au système favorisent le développement de communautés nitrifiantes, mais également de communautés bactériennes susceptibles d'assurer la dégradation de certains composés considérés comme difficilement biodégradables, dans des conditions de travail conventionnelles (CLARA et al., 2005a,b; GRASMICK et al., 2009). Cependant, des TRS importants ont tendance à accumuler de la matière inerte dans le bioréacteur au détriment des conditions d'agitation et de mélange, et des efficacités de transfert d'oxygène (LOBOS et al., 2009).

Quant au TRH, il a une influence mitigée sur l'élimination des micropolluants dans le BRM. En effet, certaines études notent que le TRH n'a pas d'influence particulière sur l'élimination des composés hydrophobes, à cause de la capacité de ces derniers à se maintenir dans le bioréacteur par

Tableau 3. Évolution de la pression transmembranaire au cours de chaque étape de filtration.

Table 3. Evolution of transmembrane pressure for each filtration step.

\begin{tabular}{|c|c|c|c|c|c|}
\hline \multirow[b]{2}{*}{ Conditions opératoires } & \multicolumn{2}{|c|}{ Étape 1} & \multicolumn{2}{|c|}{ Étape 2} & \multirow[b]{2}{*}{ Références } \\
\hline & $\begin{array}{l}\text { Durée } \\
\text { (h) }\end{array}$ & $\mathrm{kPa} \cdot \mathrm{h}^{-1}$ & $\begin{array}{l}\text { Durée } \\
\text { (h) }\end{array}$ & $\mathrm{kPa} \cdot \mathrm{h}^{-1}$ & \\
\hline 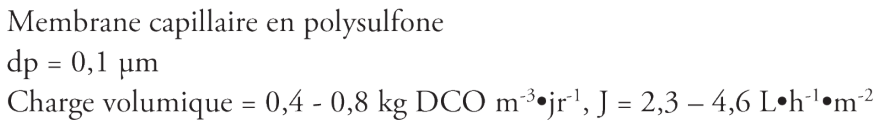 & 800 & 0,0002 & 108 & 0,27 & $\begin{array}{l}\text { ORANTES } \\
\text { et al., } 2006\end{array}$ \\
\hline 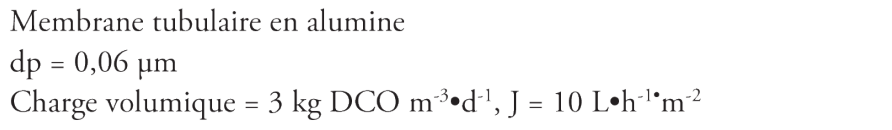 & 550 & 0,036 & 30 & 1,28 & $\begin{array}{c}\text { OGNIER et al., } \\
2004\end{array}$ \\
\hline 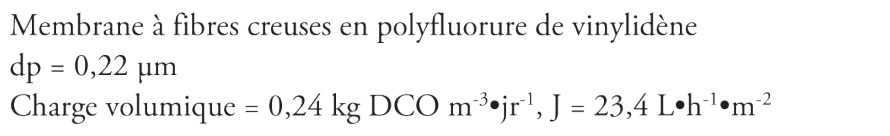 & 300 & 0,015 & 12 & 2,88 & YU et al., 2003 \\
\hline 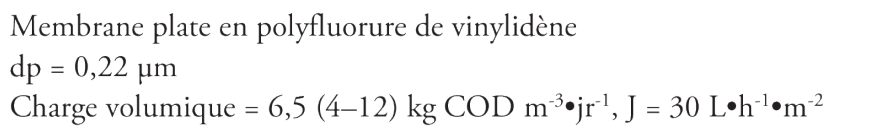 & 360 & 0,05 & 65 & 0,25 & $\begin{array}{c}\text { CHO et FANE, } \\
2002\end{array}$ \\
\hline
\end{tabular}


adsorption sur la biomasse. Par contre, le TRH a une influence sur les composés moyennement hydrophobes et hydrophiles. CHEN et al. (2008) ont observé des concentrations constantes de BPA à la sortie d'un BRM, malgré la variation du TRH (8 h à 3,9 h). HU et al. (2007) ont observé un faible taux d'abattement de l'activité ostrogénique lorsque le TRH est de $6 \mathrm{~h}$, et des taux d'abattement plus élevés et croissants lorsque le TRH est supérieur à $6 \mathrm{~h}$. La concentration élevée de biomasse dans le BRM (8 à $\left.25 \mathrm{~g} \bullet \mathrm{L}^{-1}\right)$ améliore l'élimination des polluants par biodégradation et adsorption sur la biomasse (CIRJA et al., 2008).

\subsection{3 pH et température}

Le $\mathrm{pH}$ dans le BRM a une influence sur certaines propriétés physico-chimiques des polluants. Une étude a montré que les $\mathrm{pH}$ élevés $(\mathrm{pH}>10)$ ou faibles $(\mathrm{pH}<4)$ induisent une baisse de l'hydrophobicité de certains composés tels que le norfloxacine, alors que ce dernier est très hydrophobe à des $\mathrm{pH}$ près de sept (MCBRIEN et al., 2004). Le $\mathrm{pH}$ (ou pKa) agit sur l'état protonique des composés (hydrophile ou hydrophobe). LYKO et al. (2005) ont rapporté que le BPA est adsorbé à $30 \%$ à $\mathrm{pH}=5$, mais il y a désorption à un $\mathrm{pH}$ plus élevé. Le $\mathrm{pH}$ dans le bioréacteur est un paramètre à contrôler. La température influence quant à elle la solubilité des polluants et certaines de leurs propriétés physico-chimiques telles que l'hydrophobicité et la solubilité. CARBALLA et al. (2005) ont observé une très bonne performance en matière d'élimination du dichlofénaque, naproxène et ibuprofène à $25^{\circ} \mathrm{C}$. Cependant, à $12{ }^{\circ} \mathrm{C}$, les performances observées sont faibles. CLARA et al. (2005b) ont rapporté que le BRM élimine le benzafibrate à $90 \%$ en été $\left(20^{\circ} \mathrm{C}\right)$, mais ils notent un faible taux d'élimination à $12^{\circ} \mathrm{C}$. Ces mêmes auteurs ont par ailleurs démontré que l'âge critique des boues pour l'élimination du bisphénol A (BPA) est une fonction de la température (Équation 2) (CLARA et al., 2005b).

$$
\theta_{\mathrm{T}}=\theta_{\text {Topér }} * 1,072^{(\mathrm{T}-10)}
$$

\subsubsection{Hydrophobicité et structure chimique des polluants}

Le devenir des polluants dans le BRM est influencé par des facteurs tels que les propriétés chimiques et physico-chimiques des polluants (ex. : hydrophobicité et structure chimique, etc.). Un composé hydrophile difficilement biodégradable peut se retrouver à la sortie du BRM (en un temps TRH) sans avoir été dégradé. En revanche, un composé hydrophobe difficilement biodégradable peut s'adsorber sur la biomasse et être maintenu dans le bioréacteur (GARCIA et al., 2002; GIGER et al., 2003; ILANI et al., 2005; LINDBERG et al., 2006; YU et HUANG, 2005). Le temps de contact de ce dernier dans le bioréacteur sera alors égal au TRS, ce qui augmentera et facilitera sa biodégradation. Il est important de noter que l'hydrophobicité d'un composé est exprimée par la valeur de son coefficient de partage octanol/eau " $\mathrm{K}_{\text {ow }}$ " (JAWAD, 2008). Ce coefficient " $\mathrm{K}_{\mathrm{ow}}$ " se définit comme étant le ratio de la concentration du composé dans le solvant organique (octanol) sur sa concentration dans la phase aqueuse (eau) (LYMAN, 1990). La valeur logarithmique du coefficient de partage ( $\log \mathrm{K}_{\text {ow }}$ ) est inférieure à 2,5 pour un composé qui ne s'adsorbe pas ou s'adsorbe difficilement sur la biomasse. Lorsque le composé est moyennement hydrophobe, la valeur logarithmique de son coefficient de partage est comprise entre 2,5 et 4,0. En revanche, lorsque le composé est caractérisé par un fort potentiel d'adsorption sur la biomasse, la valeur logarithmique du coefficient de partage est supérieure à 4,0 (composé très hydrophobe) (LYMAN, 1990).

La structure chimique d'un polluant pourrait influencer son élimination au cours d'un processus biologique. En effet, plus la structure chimique du polluant est complexe, moins il est biodégradable. Il est rapporté que l'augmentation des noyaux aromatiques ou des groupements toxiques $\left(\mathrm{SO}_{2} \mathrm{O}^{-}\right)$ fait décroître la dégradation dans une classe de micropolluants (ANDREOZZI et al., 2006; CIRJA et al., 2008; REEMTSMA et al., 2002). En effet, REEMTSMA et al. (2002) ont observé que le naphtalène monosulfonate est complètement éliminé dans le BRM, tandis que le naphtalène disulphonate est éliminé à $40 \%$ dans des conditions identiques d'opération. Ces auteurs ont également rapporté que la présence du groupement éthinyle dans $17 \beta$-éthinyløstradiol lui procure une aptitude de biodégradation différente et plus faible que celle observée sur le $17 \alpha$-œstradiol, malgré leurs structures de base identiques (Figure 3).

\section{COLMATAGE MEMBRANAIRE ET MODÉLISATION}

Dans le passé, le facteur limitant le développement de la technologie de BRM était le coût des membranes (MARROT et al., 2004). Cependant, depuis quelques années, les coûts ont été réduits grâce aux progrès effectués dans le domaine de la fabrication des membranes. Des progrès ont permis également d'augmenter la durée de vie des membranes, de diminuer les coûts de remplacement de ces dernières, de réduire la consommation énergétique par rapport au flux de perméat obtenu, et de diminuer les coûts de fonctionnement par les possibilités de réutilisation des eaux traitées. De nos jours, le principal facteur limitant l'application à long terme des BRM est devenu le colmatage des membranes. C'est un phénomène complexe. Il peut être décrit comme étant une diminution continue du flux de perméat (à pression constante) ou une augmentation de la PTM, causée par l'accumulation 

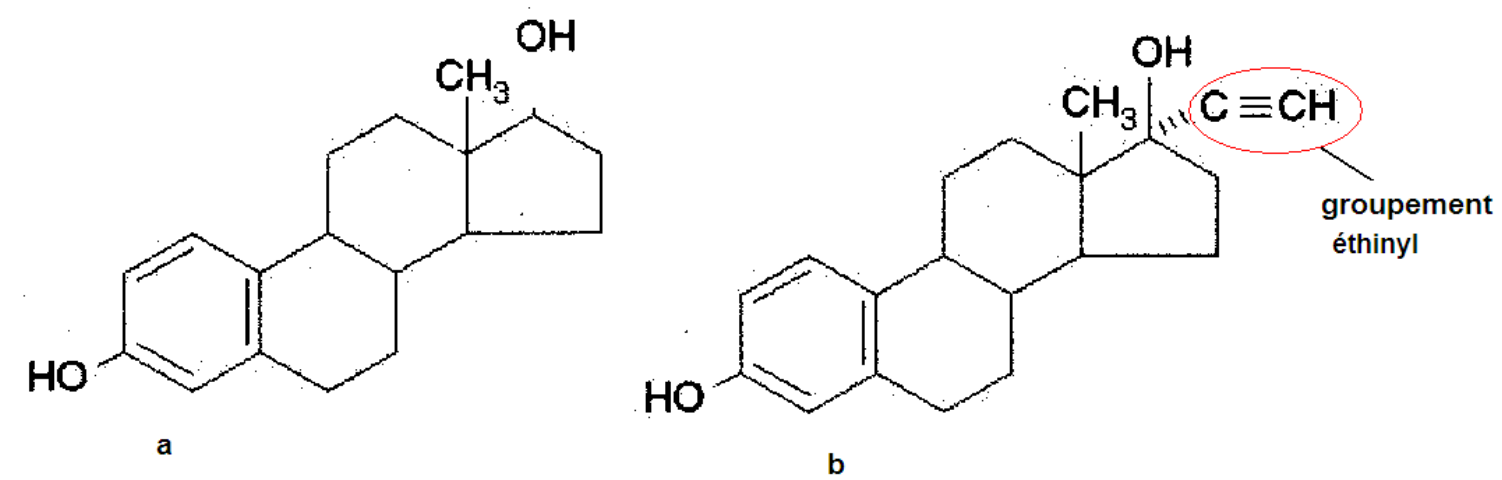

Figure 3. Structures chimiques de deux composés oestrogéniques : a) $17 \beta$-estradiol (E2), b) 17 $\alpha$-éthinylestradiol (EE2). Chemical structures of two estrogenic compounds: a) 17ß-estradiol (E2) b) 17 $\alpha$-éthinylestradiol (EE2).

ou l'adhésion et/ou l'adsorption de particules solubles ou colloïdales (organiques, inorganiques et cellules microbiennes) ou en suspension à la surface de la membrane et sur les pores de la membrane (CHOO et LEE, 1996). Il est possible de quantifier le colmatage en fonction de sa réversibilité : le colmatage réversible (de nature physique) et le colmatage irréversible (de nature plutôt chimique). Plusieurs facteurs peuvent contribuer au colmatage des membranes et la nature de ce colmatage varie d'un auteur à l'autre, alors qu'il est plutôt le reflet des conditions opératoires et de la configuration membranaire utilisée. En effet, en fractionnant le colmatage suivant trois échelles d'observation : le macrocolmatage (accumulation de MES sur la membrane), le microcolmatage (développement de biofilm sur la membrane) et le nanocolmatage (adsorption de molécules), on classera des auteurs qui observent plutôt :

- du macrocolmatage et qui soulignent l'importance de la boue activée et son rôle dans le colmatage (LI et al., 2005) et qui considèrent les MES comme la principale cause du colmatage des membranes (BRINDLE et STEPHENSON, 1996; YAMAMOTO et al., 1989). L'intérêt de ce gâteau, en tant que couche dynamique sélective, peut améliorer la capacité de rétention de la membrane (OGNIER et al., 2004; TARDIEU et al., 1999), mais deviendra vite limitant en fonction du flux de perméat (> $10 \mathrm{LMH})$;

- du microcolmatage et qui soulignent l'importance des produis microbiens solubles (PMS) générés durant les réactions biologiques et des substances polymériques extracellulaires (SPE) (DEFRANCE et al., 2000; LEE et al., 2003);

- du nanocolmatage et qui remarquent une contribution significative des composés dissous. Selon FANE et al. (1980), les substances dissoutes contribueraient jusqu'à $60 \%$ au colmatage.
Ce colmatage membranaire doit être maîtrisé et contrôlé afin de maintenir des conditions de perméabilité nécessaires à la longévité de la membrane et à la productivité du système. Des conditions opératoires et un design adaptés permettent, en effet, de minimiser le macrocolmatage (LEBEGUE et al. 2009). La maîtrise de ce colmatage conduit, entre autres, à la réduction des coûts liés aux opérations de nettoyage de la membrane.

\subsection{Paramètres influençant le colmatage membranaire}

Plusieurs études ont tenté d'élucider les mécanismes et facteurs qui influencent et gouvernent le colmatage membranaire. Même si le colmatage est le résultat de phénomènes complexes, il est considérablement influencé par les conditions opératoires, hydrodynamiques, le type et la configuration de la membrane et les caractéristiques des boues (MES, état physiologique de la biomasse, viscosité, etc.) (CHOI et al., 2005; CICEK et al., 1998; KAAM et al., 2006).

\subsubsection{Composition de la liqueur mixte}

Les caractéristiques de la biomasse présente dans le bioréacteur ont une influence significative sur le colmatage de la membrane. Il a été rapporté que le colmatage s'amplifie dès que l'état de floculation de la biomasse dans le bioréacteur est faible (KAAM et al., 2006). LI et al. (2005) ont pu réduire le macrocolmatage en développant, dans le bioréacteur, des boues granulées. Le flux de perméat obtenu dans ces conditions est deux fois plus élevé que celui observé dans un BRM classique fonctionnant dans les mêmes conditions de travail. CICEK et al. (1998) ont observé que lorsque les concentrations de MES sont comprises entre 8 et $15 \mathrm{~g} \bullet \mathrm{L}^{-1}$, le flux de perméat est plus élevé et décroît plus lentement et graduellement, comparativement aux concentrations de MES sont comprises entre 2 et $8 \mathrm{~g} \bullet \mathrm{L}^{-1}$. 


\subsubsection{Vitesse d'écoulement transversal et flux transmembranaire}

La vitesse d'écoulement transversal ou le flux transmembranaire $\left(\mathrm{v}_{\mathrm{T}}\right)$ a une influence sur le colmatage et la nature du colmatage de la membrane. CHOI et al. (2005) ont rapporté que lorsque le BRM à boucle externe opère à faibles vitesses d'écoulement transversal, c'est-à-dire entre 1 et $2 \mathrm{~m} \bullet \mathrm{s}^{-1}$ pour une membrane de UF et entre 1 et $3 \mathrm{~m} \cdot \mathrm{s}^{-1}$ pour une membrane de MF, le colmatage qui s'installe est réversible. Par contre, pour des vitesses $\geq 3 \mathrm{~m} \bullet \mathrm{s}^{-1}$, le colmatage devient irréversible. Un flux stable peut être maintenu pendant une longue période de filtration, lorsque le BRM opère à une PTM raisonnablement ajustée. De nombreuses études indiquent que le flux à travers la membrane, ou le flux transversal et la PTM doivent être maintenus inférieurs à des valeurs critiques respectives (notion de flux critique). Ces valeurs critiques doivent être déterminées pour chaque type d'effluent, de membrane et de configuration du module membranaire (CICEK et al., 1998; DEFRANCE et JAFFRIN, 1999).

\subsection{Maîtrise du colmatage}

Quelques méthodes préventives, ou l'utilisation de conditions opératoires appropriées, limitent ou réduisent le dépôt de particules à l'intérieur des pores ou sur la couche active de la membrane.

\subsubsection{Prétraitement de l'affluent}

Suivant sa composition, la suspension peut être prétraitée afin de diminuer son pouvoir colmatant. Quelques opérations, telles que la préfiltration, la dilution, la décantation, l'homogénéisation, etc., agissent sur des paramètres physiques. Il est également à noter l'avantage du prétraitement de type grille perforée par rapport au microtamis, où la rétention des fibres (ex. : cheveux, etc.) est plus faible. D'autres techniques modifient les paramètres chimiques (modification du $\mathrm{pH}$, modification de la force ionique de la solution par ajout de sels ou ajout de réactifs de coagulation, ou de floculation, ou encore, ajout de complexant, etc.) (MOULIN, 1990; POUET et al., 1992). Par exemple, le conditionnement préalable d'une suspension par voie physico-chimique (apport de coagulant) permet de favoriser la structuration des flocs et améliorer la filtrabilité de la suspension. L'utilisation de charbon actif en poudre permet de fixer les molécules solubles ou les colloïdes à l'origine du colmatage irréversible (LESAGE et al., 2005).

\subsubsection{Vitesse d'écoulement transversal et aération du bioréacteur}

La perméabilité est principalement gouvernée par la vitesse d'écoulement transversal du flux dans le BRM à boucle externe et par l'aération dans le BRM immergé. Dans ce dernier, l'injection d'air sous et à l'intérieur de la membrane permet d'atténuer la formation d'un gâteau sur la surface extérieure de la membrane (MATOSIC et al., 2008; PELLEGRIN et al., 2002). L'aération produit l'oxygène nécessaire à l'activité biologique et crée une turbulence autour de la membrane qui entraîne les grosses particules et détache le gâteau qui se forme sous l'effet de la succion requise pour la filtration (METCALF et EDDY INC., 2003). Cependant, l'aération pourrait avoir des impacts négatifs sur les performances du bioréacteur. En effet, KAAM et al. (2006) ont observé que le mode d'aération a une influence sur la physiologie de la biomasse. Selon ces auteurs, l'aération en alternance permettrait de limiter le colmatage.

\subsubsection{Nettoyage des membranes}

Le nettoyage des membranes élimine le colmatage et prolonge ainsi leur durée de vie. Il existe plusieurs techniques de nettoyage des membranes. Les plus utilisées sont le rétrolavage et le lavage chimique. Le rétrolavage se fait à l'eau ou à l'air. Plus les pores de la membrane sont larges, moins le rétrolavage est efficace. Plusieurs paramètres influencent l'efficacité du rétrolavage, notamment le débit du rétrolavage (en général, ce débit est deux à trois fois supérieur au débit de filtration), la fréquence et la durée du rétrolavage. La littérature révèle que plus le rétrolavage est de courte durée, plus il permet de rétablir un meilleur flux (BARRIOS-MARTINEZ et al., 2006; RAMIREZ et DAVIS, 1998). Comme seules les membranes de type fibres creuses pouvaient être rétrolavées (arrachement des joints de colle et gonflement des membranes planes pendant le rétrolavage), des fabricants travaillent actuellement sur la fabrication de membranes planes rétrolavables.

Le nettoyage chimique peut être fait avec différents types de produits chimiques. On utilise principalement des solutions acides pour éliminer les dépôts inorganiques, des solutions basiques et des agents tensioactifs pour éliminer les matières organiques et des désinfectants pour prévenir la croissance de micro-organismes dans le système membranaire (BOUCHARD et al., 2000). Le choix des produits chimiques se fait en fonction de la nature du matériau membranaire, afin de respecter les limites de $\mathrm{pH}$ et de température ainsi que la tolérance au chlore, aux autres désinfectants et acides. Dans les conditions normales, les solutions généralement utilisées pour le nettoyage chimique sont l'hypochlorite de sodium $(\mathrm{NaOCl})$ pour détacher les matières organiques et l'acide citrique $\left(\mathrm{C}_{6} \mathrm{H}_{8} \mathrm{O}_{7}\right)$ pour les inorganiques (CHANG et KIM, 2005; CHANG et al., 1999; LE-CLECH et al., 2006; LEE et al., 2003). Il est fréquent que les fabricants de membranes proposent des protocoles spécifiques pour le nettoyage chimique. Ces nettoyages sont, pour la plupart, opérationnels sans vider le bioréacteur où les solutions de nettoyage sont envoyées pendant un rétrolavage et présentent peu d'impacts sur le procédé biologique. Les notices d'emploi du module membranaire renferment des recommandations précises quant aux produits à utiliser pour nettoyer et désinfecter les membranes sans les détériorer. Il est 
aussi important de procéder à des désinfections périodiques $\mathrm{du}$ système (circuits/compartiments d'alimentation et de perméat) et de construire un précieux historique des membranes depuis le début de leur utilisation (base de données). Cet historique pourrait servir à ajuster les conditions opératoires et à prédire la durée de vie des membranes.

\subsection{Modélisation du colmatage}

Il existe plusieurs modèles mathématiques de description du colmatage, notamment le modèle hydrodynamique, le modèle fractal, le modèle de résistance par section et les modèles de résistances en série.

\subsubsection{Modèle hydrodynamique}

Le modèle hydrodynamique proposé par LIU et al. (2003) (cité par NG et KIM, 2007) décrit le colmatage en fonction des paramètres hydrodynamiques du BRM (vitesse d'écoulement transversal ou flux à travers la membrane, flux de perméat, intensité d'aération, concentration des MES, etc.). Dans ce modèle, le taux de croissance du colmatage $\left(\mathrm{k}, \mathrm{m} \bullet \mathrm{h}^{-1}\right)$ est décrit par l'équation 3 :

$$
\mathrm{k}=\mathrm{f}_{2} \mathrm{v}_{\mathrm{T}}^{\mathrm{c}} \mathrm{J}^{\mathrm{d}} \mathrm{X}_{\mathrm{b}}^{\mathrm{e}}
$$

Dans l'expression ci-dessus, « $\mathrm{f}_{2}$ ", « $\mathrm{c}$ », « $\mathrm{d}$ » et « $\mathrm{e}$ » sont des constantes, " $\mathrm{v}_{\mathrm{T}}, \mathrm{m} \bullet \mathrm{s}^{-1} »$ est la vitesse d'écoulement transversal ou flux, « J, $\mathrm{L} \bullet \mathrm{m}^{-2} \bullet \mathrm{h}^{-1}$ » est le flux de perméat et « $\mathrm{X}_{\mathrm{b}}, \mathrm{g} \bullet \mathrm{L}^{-1}$ » est la concentration de MES dans le bioréacteur. Les valeurs des constantes " $\mathrm{f}_{2}$ ", " $\mathrm{c}$ ", " $\mathrm{d}$ " et " $\mathrm{e}$ " sont déterminées par la méthode des moindres carrés et sont les suivantes $\mathrm{f}_{2}=8,933 \times 107, \mathrm{c}=-3,047, \mathrm{~d}=0,376$, et e $=9,532$. L'équation 3 caractérise de façon quantitative le colmatage de la membrane.

\subsubsection{Modèle fractal}

MENG et al. (2005) ont développé un modèle pour évaluer la perméabilité du gâteau à la surface de la membrane dans un BRM immergé. La microstructure désordonnée et complexe du gâteau y est décrite par la théorie fractale. Le taux de croissance du colmatage $\left(\mathrm{k}, \mathrm{m} \bullet \mathrm{h}^{-1}\right)$ est défini par l'équation 4 :

$$
\mathrm{k}=\frac{\mu^{*} \mathrm{~L}_{0} \mathrm{Q}}{\mathrm{PTM}^{*} \mathrm{~A}_{\mathrm{t}}}=\frac{\mathrm{G}}{\mathrm{g}^{2}} \mathrm{C}_{0} \frac{1}{\mathrm{~A}_{\mathrm{t}}} \frac{2-\mathrm{D}_{\mathrm{s}}}{3-\mathrm{D}_{\mathrm{s}}} \alpha_{\max }^{3-\mathrm{D}_{\mathrm{s}}}
$$

Dans l'expression ci-dessus, " $\mu, \mathrm{mPa}^{\bullet} \mathrm{s}^{-1} »$ est la viscosité dynamique de la liqueur mixte, " $\mathrm{L}_{0}$ " est une constante caractérisant la longueur de pore, « $\mathrm{Q}, \mathrm{L} \bullet \mathrm{s}^{-1}$ » est le débit à travers la membrane, "PTM, Pa » est la pression transmembranaire, " $A_{t}$ " est la surface totale des pores $\left(\mathrm{m}^{2}\right)$, " $G$ » est le facteur géométrique du flux à travers un pore (i.e. $\mathrm{Pi} / 128$ pour les pores circulaires), " $\mathrm{g}$ " est le facteur de forme, " $\mathrm{C}_{0}$ " est une constante, " $\mathrm{D}_{\mathrm{s}}$ " est la dimension de la surface du pore selon la théorie fractale, " $a_{\max }$ " est la limite surfacique maximale d'un pore. Le modèle n'indique pas l'influence des paramètres et conditions opératoires sur la résistance du gâteau.

\subsubsection{Modèle de résistance par section}

LI et WANG (2006) ont utilisé une approche de résistance par section pour décrire le colmatage dans un BRM. Dans le modèle proposé, la surface de la membrane est divisée en plus petites surfaces identiques. Chaque petite surface a une résistance intrinsèque, une résistance de colmatage des pores, une résistance d'accumulation de gâteaux et une résistance de formation de couches dynamiques. Les dynamismes d'attachement et de détachement de la biomasse à la surface membranaire sont pris en compte, et la résistance totale $\left(R_{T}\right)$ est la somme de toutes les résistances : résistances intrinsèques $\left(R_{m}\right)$, résistances de colmatage des pores $\left(R_{c}\right)$, résistances d'accumulation du gâteau $\left(\mathrm{R}_{\mathrm{AG}}\right)$ et résistances de formation de couches dynamiques $\left(\mathrm{R}_{\mathrm{CD}}\right)$ (Équation 5):

$$
\mathrm{R}_{\mathrm{T}}=\mathrm{R}_{\mathrm{m}}+\mathrm{R}_{\mathrm{c}}+\mathrm{R}_{\mathrm{AG}}+\mathrm{R}_{\mathrm{CD}}
$$

La masse de boue sur la couche dynamique de colmatage $\left(\mathrm{M}_{\mathrm{CD}}\right)$ est exprimée par l'équation 6 :

$$
\frac{\mathrm{dM}_{\mathrm{CD}}}{\mathrm{dt}}=-\frac{24 * \mathrm{X}_{\mathrm{b}}^{*} \mathrm{~J}^{2}}{24^{*} \mathrm{~J}+\mathrm{C}_{\mathrm{d}}{ }^{*} \mathrm{~d}_{\mathrm{p}}{ }^{*} \mathrm{I}}-\beta^{*} \frac{(1-\alpha)^{*} \mathrm{I}^{*} \mathrm{M}_{\mathrm{CD}}^{2}}{\mathrm{Y}_{\mathrm{b}}{ }^{*} \mathrm{~V}_{\mathrm{p}}{ }^{*} \mathrm{t}_{\mathrm{f}}+\mathrm{M}_{\mathrm{CD}}}
$$

Le premier terme de cette expression décrit le taux d'attachement de la biomasse et le deuxième terme est le taux de détachement de la biomasse. " $\mathrm{X}_{\mathrm{b}}$ " est la concentration de biomasse $\left(\mathrm{g} \bullet \mathrm{L}^{-1}\right)$, « J, $\mathrm{L} \bullet \mathrm{m}^{-2} \bullet \mathrm{s}^{-1}$ » est le flux du perméat, « $\mathrm{C}_{\mathrm{d}}$ " est le coefficient de la force de levée d'une particule de boue de diamètre « $d_{p}, m$ ", " $\beta$ » est le coefficient du taux d'érosion de la biomasse dynamique, "I » est l'intensité de cisaillement sur la surface de la membrane, " $V_{P}$, $\mathrm{m}^{3}$ » est le volume de perméat au cours d'un cycle de filtration, " $\mathrm{Y}_{\mathrm{b}}$ " est le coefficient de compression de la boue, " $t_{\mathrm{f}}$ sec $"$ est le temps de filtration. $\mathrm{M}_{\mathrm{CD}}$ s'exprime en $(\mathrm{g})$. Le modèle de résistance par section intègre les effets de variation des forces de cisaillement sur la formation de gâteaux et a l'avantage de prendre en compte les cycles de nettoyage. Il caractérise le colmatage en tout temps. Cependant, les limites de ce modèle résident dans le fait que les paramètres biologiques sont considérés comme constants. 


\subsubsection{Modèles de résistance en série}

Les modèles de résistance en série sont des modèles qui intègrent les effets de la biomasse aux processus de colmatage de la membrane. Les modèles de résistance en série prennent en compte, en plus des MES, les effets des matières solubles dans le processus de colmatage. Dans ces modèles, le colmatage réversible est attribué aux MES, tandis que les matières solubles sont responsables du colmatage irréversible. Le flux du perméat est décrit selon l'équation de Darcy, donnée à l'équation 7 :

$$
\mathrm{J}=\frac{\mathrm{PTM}}{\mu^{*} \mathrm{R}_{\mathrm{T}}}
$$

Dans cette expression, " $\mathrm{J}, \mathrm{m} \bullet \mathrm{s}^{-1}$ " représente le flux du perméat, "PTM, Pa » est la pression transmembranaire, " $\mu$, Pa.s » est la viscosité de la liqueur mixte et « $\mathrm{R}_{\mathrm{T}}, \mathrm{m}^{-1}$ " est la résistance totale de la membrane. L'outil le plus simple et le plus employé sur le plan pratique pour représenter l'évolution du colmatage en cours d'opération est le modèle de résistance en séries (GRASMICK et al., 2009), modèle selon lequel la résistance totale de la membrane $\left(\mathrm{R}_{\mathrm{T}}\right)$ peut être définie comme suit :

$$
\mathrm{R}_{\mathrm{T}}=\mathrm{R}_{\mathrm{m}}+\mathrm{R}_{\mathrm{rev}}+\mathrm{R}_{\text {irr }}
$$

Dans cette expression, " $\mathrm{R}_{\mathrm{m}}$ " représente la résistance hydraulique initiale de la membrane, " $\mathrm{R}_{\mathrm{rev}}$ " est la résistance réversible et " $\mathrm{R}_{\mathrm{irr}}$ ", la résistance irréversible. Certains auteurs ont adopté une description plus simplifiée (CHOI et al., 2005; LIANG et al., 2006; ZHANG et al., 2006).

D'autres ont établi des modèles qui intègrent l'influence des PMS. C'est dans cet ordre que LEE et al. (2002) ont proposé l'expression suivante pour la résistante totale (Équation 9):

$$
\mathrm{R}_{\mathrm{T}}=\mathrm{R}_{\mathrm{m}}+\mathrm{m}^{*} \alpha
$$

Dans cette expression, " $\mathrm{R}_{\mathrm{m}}$ " est la résistance propre de la membrane, " $\alpha$ » est la résistance spécifique et " $\mathrm{m}$, $\mathrm{g}$ ", un facteur estimé par l'équation 10 :

$$
\mathrm{m}=\mathrm{k}_{\mathrm{m}} * \mathrm{~V}_{\mathrm{p}} * \frac{\mathrm{X}_{\mathrm{b}}}{\mathrm{A}}
$$

Avec $« \mathrm{k}_{\mathrm{m}}$ ", le coefficient qui reflète les effets de filtration $\left(\mathrm{k}_{\mathrm{m}}\right.$ est compris entre zéro et un, et est égal à un pour la filtration frontale), " $V, m^{3}$ " le volume de perméat, " $X_{b}$, $\mathrm{g} \bullet \mathrm{L}^{-1}$ » la concentration de $\mathrm{MES}$ et " $\mathrm{A}, \mathrm{m}^{2}$ » la surface active de la membrane. WINTGENS et al. (2003) ont proposé un autre modèle qui associe l'activité biologique au colmatage, mais ce modèle ne quantifie pas les substances. Le flux de perméat est décrit en fonction de la PTM selon l'équation 11:

$$
\mathrm{J}=\frac{\Delta \mathrm{PTM}}{\mu^{*}\left(\mathrm{R}_{\mathrm{m}}+\mathrm{R}_{\mathrm{g}}+\mathrm{R}_{\mathrm{c}}\right)}
$$

Dans cette expression, " $\mathrm{R}_{\mathrm{m}}$ " est la résistance hydraulique initiale de la membrane, " $\mathrm{R}_{\mathrm{g}}$ " est la résistance du gâteau, " $\mathrm{R}_{\mathrm{c}}$ " est la résistance de colmatage et " $\mu, \mathrm{mPa}^{\bullet} \mathrm{s}^{-1}$ " est la viscosité de la liqueur mixte. " $\triangle \mathrm{PTM}, \mathrm{Pa}$ » est la différence de pression transmembranaire effective et elle est obtenue à partir de l'équation 12 :

$$
\Delta \mathrm{PTM}=\mathrm{p}_{\text {hydro }}+\mathrm{p}_{\mathrm{pomp}}-\Delta \mathrm{p}_{\mathrm{ax}}
$$

Dans cette équation, " $\mathrm{p}_{\text {hydro' }} \mathrm{Pa}$ " est la pression hydrostatique, " $\mathrm{p}_{\text {pomp }}, \mathrm{Pa}$ " est la pression de succion, " $\Delta \mathrm{p}_{\mathrm{ax}}$, $\mathrm{Pa}$ » est la chute de pression du flux de perméat. Il faut retenir que la complexité du colmatage limite sa description mathématique. Les différents modèles établis à ce jour ne font pas une description complète et intégrale de l'évolution du colmatage en cours d'opération.

\subsection{Spécificité et performance du BRM}

Le BRM présente de nombreux avantages. Ce sont, notamment, les performances intéressantes en matière d'élimination de polluants biodégradables et de polluants considérés comme difficilement biodégradables dans les conditions de travail conventionnelles. La sélectivité remarquable de la membrane a pour avantages (BRINDLE et STEPHENSON, 1996; GRASMICK et al., 2009; NAGANO et al., 1992; XING et al., 2000) : 1) une grande qualité de l'eau traitée en matière de particules (absence totale de MES et de matière colloïdale); 2) une désinfection poussée dont l'intensité dépend du seuil de coupure et de la distribution des diamètres des pores des membranes; 3) une rétention totale des espèces biologiques, même peu floculées, qui peut favoriser le développement d'espèces et d'activités spécifiques au sein $\mathrm{du}$ réacteur; 4) une rétention par la membrane des MES non décantables dont le temps de séjour dans le système devient égal au temps de rétention de la phase solide $(\theta)$, facilitant ainsi leur assimilation; et 5) le maintien dans le réacteur d'une teneur contrôlée et élevée en biomasse qui permet une intensification des processus biologiques. 
La concentration en biomasse dans le BRM n'est pas limitée à une valeur critique proche de 4 à $5 \mathrm{~g} \bullet \mathrm{L}^{-1}$, valeur audelà de laquelle la séparation par décantation gravitaire apparaît comme fortement ralentie, voire perturbée, dans les systèmes conventionnels à boues activées. Le BRM est ainsi susceptible d'opérer avec des concentrations deux à cinq fois plus élevées (entre 8 et $\left.25 \mathrm{~g} \bullet \mathrm{L}^{-1}\right)$. L'effluent traité est d'une excellente qualité en matière d'abattement de la turbidité, de MES, de DBO, de $\mathrm{DCO}$, de désinfection et de sous-produits de désinfection. Cette eau traitée est complètement acceptable pour une réutilisation directe au niveau municipal (eau de toilette, lavage d'auto, etc.) ou pour une réutilisation indirecte au niveau industriel (eau de refroidissement, eau de procédé, etc.). Elle pourrait également servir d'eau d'alimentation aux unités d'osmose inverse (XING et al., 2000). Le surcoût d'investissement et de fonctionnement d'un BRM est alors compensé par la minimisation de son emprise au sol (d'un facteur quatre par rapport à un procédé boue activée conventionnelle) et le fait que la qualité de l'eau produite dispense d'un traitement tertiaire de type oxydation ou infiltration/percolation.

\subsubsection{Elimination de polluants de type organique}

En général, les taux d'abattement de la pollution de type organique dans les eaux usées sont très intéressants. Le BRM est très robuste aux variations inopinées de charges et pourrait fonctionner à charges volumiques et organiques importantes. ZHANG et VERSTRAETE (2002) ont observé, dans un BRM, un abattement de $99 \%$ de DCO sur une eau usée de charge organique de l'ordre de $10500 \mathrm{mg} \mathrm{DCO} \bullet \mathrm{L}^{-1}$. Pour une même charge volumique à traiter, le BRM permet de réduire la taille du volume réactionnel. Il permet de supprimer le décanteur secondaire, et donc d'empêcher tout « lessivage » du réacteur biologique. Il permet également de supprimer tout problème de décantabilité des boues qui, malheureusement, peut échapper ponctuellement aux opérateurs du fait de la variabilité de la composition de l'intrant ou de l'apparition de conditions de réaction non optimales, entraînant une défloculation ou l'apparition de flocs à faible décantabilité, causant un foisonnement des boues (GRASMICK et al., 2009). La production de biomasses, ou boues excédentaires, est moins importante lorsqu'on connaît les difficultés liées à la disposition de celles-ci (GANDER et al., 2000; MARROT et al., 2004).

Plusieurs études rapportent des taux d'abattement de DCO allant jusqu'à $99 \%$, dépendamment du type d'eau traitée, de composés organiques, du type de membrane et des conditions de traitement imposées (PTM, $\theta$, etc.) (Tableau 4). Par exemple, CICEK et al. (1998), en appliquant la technique de BRM (membrane céramique, seuil de coupure de $300 \mathrm{kDa}$ ) à un effluent synthétique contenant de la caséine, ont obtenu une élimination quasi totale de la DCO (99\% d'abattement) et du COD (99,5\% d'abattement), en imposant un TRH de six heures, un $\theta$ de 30 jours, une PTM de $50 \mathrm{kPa}$ et une concentration de MES comprise entre 8 et $15 \mathrm{~g} \bullet \mathrm{L}^{-1}$. En comparaison, l'application du procédé BRM mettant en jeu une membrane à fibres creuses (membrane organique, diamètre des pores de $0,04 \mu \mathrm{m})$ a permis d'obtenir un taux d'élimination de la DCO de $69 \%$ à $83 \%$ d'un effluent de lisier de porc (susceptible de contenir plusieurs molécules organiques complexes) en imposant un TRH de six heures, un TRS de 60 jours, et une concentration de MES se situant entre 2,8 et $4,1 \mathrm{~g} \bullet \mathrm{L}^{-1}$ (YANG et CICEK, 2008). Le lisier de porc issu des porcheries contient très souvent des antibiotiques (tétracycline, chlorotétracycline, etc.), lesquels sont couramment administrés aux animaux pour leur protection et leur croissance accélérée (CHEE-SANFORD et al., 2001). Ces composés sont difficilement biodégradables.

\subsection{2 Élimination des polluants de type inorganique}

D'autre part, les BRM peuvent être employés pour éliminer l'azote $\left(\mathrm{N}-\mathrm{NH}_{4}\right.$ et N-NTK) et le phosphore ( $\mathrm{P}-\mathrm{PO}_{4}$ et $\mathrm{P}_{\mathrm{T}}$ ) (Tableau 5). Il est alors possible d'utiliser le débit de recirculation du bassin membranaire (300\% à $400 \%)$ pour renvoyer en tête les nitrates produits pendant la phase aérobie. Des taux d'élimination de $\mathrm{P}_{\mathrm{T}}$ et $\mathrm{N}-\mathrm{NH}_{4}$ allant jusqu'à $97 \%$ et $99 \%$ peuvent être respectivement obtenus, dépendamment des conditions opératoires et du type d'effluent traité. BARRIOS-MARTINEZ et al. (2006), en appliquant un procédé de BRM (membrane de type inorganique) à une solution synthétique, obtiennent une élimination de $93 \%$ de $\mathrm{N}-\mathrm{NH}_{4}$ et un retrait de $90 \%$ de $\mathrm{P}-\mathrm{PO}_{4}$ en appliquant un TRH de cinq heures, un TRS de sept jours et une concentration de MES de $10 \mathrm{~g} \bullet \mathrm{L}^{-1}$. PELLEGRIN et al. (2002) ont, pour leur part, étudié la possibilité d'éliminer l'azote ammoniacal et l'azote total des eaux usées de type municipal en utilisant un BRM (membrane à fibres creuses, seuil de coupure $200 \mathrm{kDa}$ ) fonctionnant avec un TRH de six heures, un TRS de 25 jours et une concentration en MES de $11 \mathrm{~g} \bullet \mathrm{L}^{-1}$. Ils obtiennent un abattement de $95 \%$ de $\mathrm{N}^{-\mathrm{NH}_{4}}$ et de $80 \%$ de N-NTK. Les taux d'abattement de $\mathrm{N}_{-} \mathrm{NH}_{4}$ enregistrés par BARRIOS-MARTINEZ et al. (2006) et PELLEGRIN et al. (2002) sont quasiment identiques. Cependant, l'application du BRM sur des EUM requiert un $\theta$ plus élevé ( 25 jours), compte tenu de la complexité de l'effluent, comparativement à l'effluent synthétique ( $\theta$ de sept jours) employé par BARRIOS-MARTINEZ et al. (2006).

Dans un BRM conventionnel, la dénitrification est due à la présence de zones anoxies causée par les fortes concentrations de biomasse. En effet, à faible concentration d'oxygène dissous, les limites de diffusion peuvent créer, dans le bassin aérobie, des zones anoxies entre les flocs biologiques, favorisant ainsi la dénitrification (POCHANNA et al., 1999). Cependant, les processus de nitrification et dénitrification simultanés nécessitent la prise en compte de certaines conditions de 
fonctionnement et de configuration du BRM. En effet, HOLAKOO et al. (2007) ont observé, dans une étude de faisabilité de la nitrification et dénitrification simultanée dans le BRM aéré en continu, que le taux d'abattement de l'azote total n'est pas élevé (32\% à $45 \%$ ), malgré le taux élevé d'abattement de la matière organique (95\%). Il en ressort que le type d'aération peut avoir un effet significatif sur la croissance et le développement des bactéries responsables de la nitrification et la dénitrification. La concentration d'oxygène dissous nécessaire pour une oxydation du carbone et une nitrification simultanée doit être supérieure ou égale à $2 \mathrm{mg} \mathrm{O} \bullet_{2} \mathrm{~L}^{-1}$ (SORENSEN et JORGENSEN, 1993). L'aération par intermittence peut permettre d'atteindre cette concentration. L'élimination de l'azote peut également être améliorée avec la création d'une zone anoxie séparée de la zone aérobie (COTE et al., 1997). Ce type de configuration du BRM (configuration anoxie-aérobie) a permis à BAEK et PAGILLA (2008) d'obtenir des taux d'abattement de $\mathrm{N}-\mathrm{NH}_{4}$ et de $\mathrm{N}_{\mathrm{T}}$ de $97 \%$ et $89 \%$, respectivement. BIRIMA et al. (2005) ont noté, pour cette même configuration, des valeurs comparables $\left(98,2 \%\right.$ à 99,9 \% d'élimination de $\left.\mathrm{N}-\mathrm{NH}_{4}\right)$. En général, les performances du BRM en matière d'élimination biologique du phosphore sont faibles. VERA et al. (1997) ont observé un taux d'abattement de $45 \%$ de phosphore, par voie biologique. Une étude réalisée par BATTISTONI et al. (2006) rapporte une élimination de $67 \%$ du phosphore. Par contre, CICEK et al. (1998) ont mesuré un taux d'abattement de $96,6 \%$ du phosphore. Le BRM aussi est une barrière totale pour les MES

Tableau 4. Polluants organiques dégradés par les BRM.

Table 4. Organic pollutants degraded by MBR.

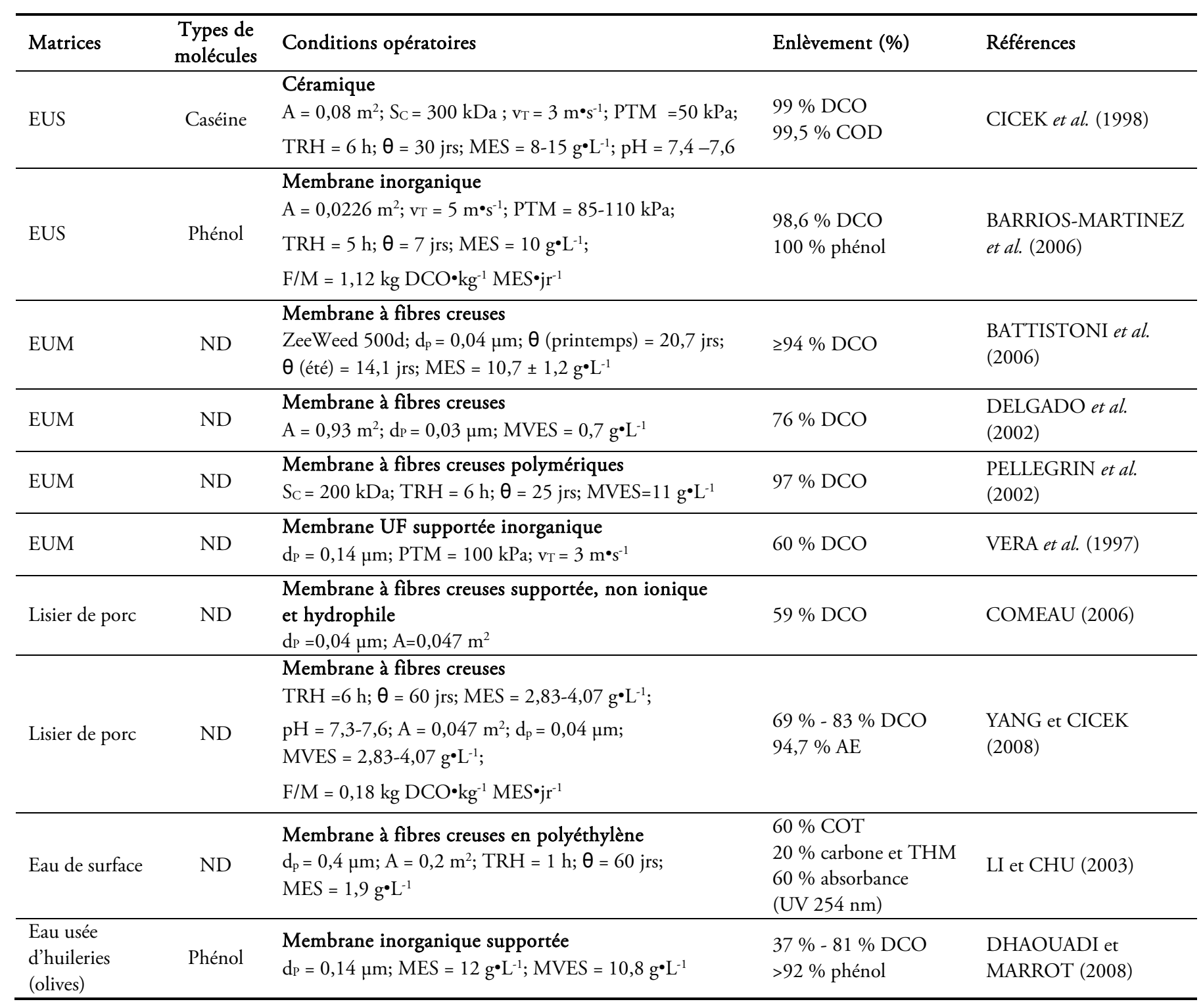


Tableau 5. Polluants inorganiques éliminés par les BRM.

Table 5. Inorganic pollutants removed by $M B R$.

\begin{tabular}{|c|c|c|c|}
\hline Matrices & Conditions opératoires & Enlèvement (\%) & Références \\
\hline Lisier de porc & $\begin{array}{l}\text { Membrane à fibres creuses } \\
\text { TRH }=8 \text { h; } \theta=60 \mathrm{jrs} ; \mathrm{MES}=2,83-4,07 \mathrm{~g} \cdot \mathrm{L}^{-1} ; \\
\mathrm{pH}=7,3-7,6\end{array}$ & $\begin{array}{l}99,9 \% \mathrm{~N}-\mathrm{NH}_{4} \text { avec contrôle } \\
\text { du pH par } \mathrm{Na}_{2} \mathrm{CO}_{3}\end{array}$ & YANG et al. (2008) \\
\hline EUS & $\begin{array}{l}\text { Membrane inorganique } \\
\mathrm{A}=0,0226 \mathrm{~m}^{2} ; \mathrm{vT}=5 \mathrm{~m}^{-1} \mathrm{~s}^{-1} \mathrm{PTM}=85-110 \mathrm{kPa} ; \\
\text { TRH }=5 \mathrm{~h} ; \theta=7 \mathrm{jrs} ; \mathrm{MES}=10 \mathrm{~g} \cdot \mathrm{L}^{-1}\end{array}$ & $\begin{array}{l}93,3 \% \mathrm{~N}^{-\mathrm{NH}_{4}} \\
89 \%-90 \% \mathrm{P}^{-\mathrm{PO}_{4}}\end{array}$ & $\begin{array}{l}\text { BARRIOS-MARTINEZ et al. } \\
\text { (2006) }\end{array}$ \\
\hline EUM & $\begin{array}{l}\text { Membrane UF inorganique supportée } \\
d_{\mathrm{P}}=0,14 \mu \mathrm{m} ; \mathrm{PTM}=100 \mathrm{kPa} ; \mathrm{v}_{\mathrm{T}}=3 \mathrm{~m} \cdot \mathrm{s}^{-1}\end{array}$ & $45 \% \mathrm{P}_{\mathrm{T}}$ & VERA et al. (1997) \\
\hline EUS & $\begin{array}{l}\text { Céramique } \\
\mathrm{A}=0,08 \mathrm{~m}^{2} ; \mathrm{S}_{\mathrm{C}}=300 \mathrm{kDa} ; \mathrm{v}_{\mathrm{T}}=3 \mathrm{~m} \cdot \mathrm{s}^{-1} ; \mathrm{PTM}=50 \mathrm{kPa} ; \\
\mathrm{TRH}=6 \mathrm{~h} ; \theta=30 \mathrm{jrs} ; \mathrm{MES}=8-15 \mathrm{~g}^{\circ} \mathrm{L}^{-1} ; \mathrm{pH}=7,4-7,6\end{array}$ & $\begin{array}{l}\geq 99,5 \% \mathrm{NTK} \\
\geq 99 \% \mathrm{~N}_{\mathrm{N} H} \\
96,6 \% \mathrm{P}_{\mathrm{T}}\end{array}$ & CICEK et al. (1998) \\
\hline EUM & $\begin{array}{l}\text { Membrane à fibres creuses polymériques } \\
\mathrm{S}_{\mathrm{C}}=200 \mathrm{kDa} ; \mathrm{TRH}=6 \mathrm{~h} ; \theta=25 \mathrm{jrs} ; \mathrm{MVES}=11 \mathrm{~g}^{\circ} \mathrm{L}^{-1} ; \\
\mathrm{MES}=11 \mathrm{~g} \cdot \mathrm{L}^{-1} ; \\
\text { Rapport F/M }=0,08 \mathrm{~kg} \text { DCO } \bullet \mathrm{kg}^{-1} \mathrm{MES} \cdot \mathrm{jr}^{-1}\end{array}$ & $\begin{array}{l}80 \% \mathrm{NTK} \\
95 \% \mathrm{~N}^{-\mathrm{NH}_{4}}\end{array}$ & PELLEGRIN et al. (2002) \\
\hline EUM & $\begin{array}{l}\text { Zenon ZeeWeed500d : membrane à fibres creuses } \\
\text { immergée } \\
d_{p}=0,04 \mu \mathrm{m} ; \theta \text { (printemps) }=20,7 \text { jrs; } \theta \text { (été) }=14,1 \text { jrs; } \\
M E S=10,7 \pm 1,2 \mathrm{~g} \cdot \mathrm{L}^{-1}\end{array}$ & $67 \% \mathrm{P}_{\mathrm{T}}$ & BATTISTONI et al. (2006) \\
\hline
\end{tabular}

( $\geq 99,9 \%)$, turbidité ( $\geq 90 \%)$ et agents pathogènes (100\%) (BARRIOS-MARTINEZ et al., 2006; CICEK et al., 1998; VERA et al., 1997) (Tableau 6).

\subsection{3 Élimination des polluants de type microbien}

L'efficacité désinfectante des BRM a été évaluée. L'application du BRM permet une élimination efficace des indicateurs de pathogènes (coliformes totaux et fécaux), des virus et des bactéries hétérotrophes aérobies (BHA) (Tableau 7). COMEAU (2006), en appliquant le BRM (membrane à fibres creuses, diamètre des pores de $0,04 \mu \mathrm{m}$ ) à un effluent de lisier de porc, a obtenu une élimination totale des coliformes fécaux (100\% d'abattement) en imposant un TRH de 9 heures. Une éradication totale de virus MS-2 (bactériophage) et de BHA a été enregistrée par CICEK et al. (1998) lors du traitement d'une EUS à l'aide d'un BRM (membrane céramique, seuil de coupure à $300 \mathrm{kDa}$ ) fonctionnant avec un TRH de 6 heures. L'efficacité désinfectante des BRM est en grande partie liée à la taille des pores. La taille des pores n'étant jamais uniforme, il faut choisir une membrane avec un écart suffisant par rapport aux plus petits micro-organismes que l'on veut retenir. Cependant, le microcolmatage réduit le seuil de coupure des membranes et certaines membranes, avec un seuil de coupure de $0,4 \mu \mathrm{m}$, présentent un abattement bactériologique total.

\subsection{4 Élimination des perturbateurs endocriniens (PE)}

Les perturbateurs endocriniens (PE) sont à l'origine de nombreuses perturbations de la faune aquatique et constituent un risque pour la santé humaine (ESPLUGAS et al., 2007; GERECKE et al., 2002; TIXIER et al., 2003). Ils échappent, pour la plupart, au traitement classique des eaux usées. Les techniques de séparation, telles que l'adsorption sur charbon actif et la filtration membranaire, peuvent être efficacement utilisées pour éliminer les PE (CHANG et al. 2009). En général, le charbon actif élimine les PE non polaires, spécialement ceux dont les valeurs de $\log \mathrm{K}_{\mathrm{ow}}$ sont supérieures à deux (RACZ et GOEL, 2010; SNYDER et al., 2003), $\mathrm{K}_{\text {ow }}$ représentant le coefficient de partage octanol/eau. Il est important de souligner que l'hydrophobicité d'un composé est exprimée par la valeur de son coefficient de partage, qui se définit comme étant le rapport entre la concentration de ce composé dans le solvant organique (octanol) et celle mesurée dans la phase aqueuse. Il est d'autant plus hydrophobe que la valeur logarithmique du coefficient de partage ( $\log \mathrm{K}_{\mathrm{ow}}$ ) est importante (LYMAN, 1990). Les techniques de filtration membranaires telles que la nanofiltration et l'osmose inverse, peuvent permettre d'atteindre $10 \%$ à $95 \%$ d'élimination des PE, dépendamment du type de PE (CHANG et al., 2009). Les performances de ces techniques membranaires sont tributaires des propriétés physicochimiques des PE, de leur taille moléculaire, de leur solubilité dans l'eau et de leurs propriétés électrostatiques (LIU et al., 2009). Les procédés d'oxydation avancée (ex. : $\mathrm{H}_{2} \mathrm{O}_{2} / \mathrm{Fe}^{2+} ; \mathrm{H}_{2} \mathrm{O}_{2} / \mathrm{O}_{3}, \mathrm{H}_{2} \mathrm{O}_{2} / \mathrm{UV}, \mathrm{O}_{3} / \mathrm{UV}$, etc.) peuvent être également utilisés pour éliminer les PE (GOGATE et PANDIT, 2004; IKEHATA et EL-DIN, 2006; PARSONS, 2004; ZAVISKA et al., 2009). Les performances de ces techniques sont généralement proportionnelles à la dose d'oxydant employée. Les études portant sur l'élimination 
Tableau 6. Élimination de solides par les BRM.

Table 6. Solids removal by MBR.

\begin{tabular}{|c|c|c|c|}
\hline Matrices & Conditions opératoires & Enlèvement (\%) & Références \\
\hline EUM & $\begin{array}{l}\text { Membrane UF inorganique supportée } \\
\mathrm{d}_{\mathrm{P}}=0,14 \mu \mathrm{m} ; \mathrm{PTM}=100 \mathrm{kPa} ; \mathrm{v}_{\mathrm{T}}=3 \mathrm{~m} \cdot \mathrm{s}^{-1}\end{array}$ & $\begin{array}{l}100 \% \text { MES } \\
92 \% \text { turbidité }\end{array}$ & VERA et al. (1997) \\
\hline Eau de surface & $\begin{array}{l}\text { Membrane à fibres creuses en polyéthylène } \\
d_{P}=0,4 \mu \mathrm{m} ; A=0,2 \mathrm{~m}^{2} ; \mathrm{TRH}=1 \mathrm{~h} ; \theta=60 \mathrm{jrs} ; \\
M E S=1,9 \mathrm{~g} \cdot \mathrm{L}^{-1}\end{array}$ & $98 \%$ turbidité & LI et $C H U(2003)$ \\
\hline EUS & $\begin{array}{l}\text { Céramique } \\
\mathrm{A}=0,08 \mathrm{~m}^{2} ; \mathrm{S}_{\mathrm{C}}=300 \mathrm{kDa} ; \mathrm{vT}=3 \mathrm{~m} \bullet \mathrm{s}^{-1} ; \mathrm{PTM}=50 \mathrm{kPa} ; \\
\mathrm{TRH}=6 \mathrm{~h} ; \theta=30 \mathrm{jrs} ; \mathrm{MES}=8-15 \mathrm{~g} \cdot \mathrm{L}^{-1} ; \mathrm{pH}=7,4-7,6\end{array}$ & $\begin{array}{l}99,9 \% \text { MES } \\
99 \% \text { turbidité }\end{array}$ & CICEK et al. (1998) \\
\hline EUS & $\begin{array}{l}\text { Membrane inorganique supportée } \\
\mathrm{A}=0,0226 \mathrm{~m}^{2} ; \mathrm{PTM}=85-100 \mathrm{kPa} ; \mathrm{TRH}=5 \mathrm{~h} ; \theta=7 \mathrm{jrs}\end{array}$ & $\begin{array}{l}100 \% \text { MES } \\
\text { turbidité < } 5 \text { NTU }\end{array}$ & $\begin{array}{l}\text { BARRIOS-MARTINEZ et al. } \\
(2006)\end{array}$ \\
\hline Lisier de porc & $\begin{array}{l}\text { Membrane à fibres creuses supportée non ionique et } \\
\text { hydrophile } \\
\mathrm{dP}=0,04 \mu \mathrm{m} ; \mathrm{A}=0,047 \mathrm{~m}^{2} ; \mathrm{TRH}=9 \mathrm{~h}\end{array}$ & $\begin{array}{l}100 \% \text { MES } \\
100 \% \text { turbidité }\end{array}$ & COMEAU (2006) \\
\hline
\end{tabular}

Tableau 7. Polluants microbiens éliminés par les BRM.

Table 7. Microbial pollutants removed by MBR.

\begin{tabular}{|c|c|c|c|}
\hline Matrices & Conditions opératoires & Enlèvement (\%) & Références \\
\hline EUM & $\begin{array}{l}\text { Membrane UF inorganique supportée } \\
\mathrm{d}_{\mathrm{P}}=0,14 \mu \mathrm{m} ; \mathrm{PTM}=100 \mathrm{kPa} ; \mathrm{v}_{\mathrm{T}}=3 \mathrm{~m} \cdot \mathrm{s}^{-1}\end{array}$ & $100 \%$ CT & VERA et al. (1997) \\
\hline Eau de surface & $\begin{array}{l}\text { Membrane à fibres creuses en polyéthylène } \\
\mathrm{d}_{\mathrm{P}}=0,4 \mu \mathrm{m} ; \mathrm{A}=0,2 \mathrm{~m}^{2} ; \mathrm{TRH}=1 \mathrm{~h} ; \\
\theta=60 \mathrm{jrs} ; \mathrm{MES}=1,9 \mathrm{~g} \cdot \mathrm{L}^{-1}\end{array}$ & 4 à 5 unités $\log$ CT & LI et CHU (2003) \\
\hline EUS & $\begin{array}{l}\text { Céramique } \\
\mathrm{A}=0,08 \mathrm{~m}^{2} ; \mathrm{S}_{\mathrm{C}}=300 \mathrm{kDa} ; \mathrm{v}_{\mathrm{T}}=3 \mathrm{~m} \cdot \mathrm{s}^{-1} ; \\
\mathrm{PTM}=50 \mathrm{kPa} ; \mathrm{TRH}=6 \mathrm{~h} ; \theta=30 \mathrm{jrs} ; \\
\mathrm{MES}=8-15 \mathrm{~g}^{\bullet} \mathrm{L}^{-1} ; \mathrm{pH}=7,4-7,6\end{array}$ & $\begin{array}{l}100 \% \text { virus MS-2 (bactériophage) } \\
100 \% \text { BHA }\end{array}$ & CICEK et al. (1998) \\
\hline EUS & $\begin{array}{l}\text { Membrane inorganique supportée } \\
\mathrm{A}=0,0226 \mathrm{~m}^{2} ; \mathrm{PTM}=85-100 \mathrm{kPa} \\
\mathrm{TRH}=5 \mathrm{~h} ; \theta=7 \mathrm{jrs}\end{array}$ & $100 \%$ micro-organismes & $\begin{array}{l}\text { BARRIOS-MARTINEZ et al. } \\
(2006)\end{array}$ \\
\hline Lisier de porc & $\begin{array}{l}\text { Membrane à fibres creuses supportée, non ionique } \\
\text { et hydrophile } \\
\mathrm{d}_{\mathrm{P}}=0,04 \mu \mathrm{m} ; \mathrm{A}=0,047 \mathrm{~m}^{2} ; \mathrm{TRH}=9 \mathrm{~h}\end{array}$ & $100 \% \mathrm{CF}$ & COMEAU (2006) \\
\hline $\begin{array}{l}\text { CF : } \\
\text { CT : } \\
\text { BHA : }\end{array}$ & $\begin{array}{l}\text { Coliformes fécaux } \\
\text { Coliformes totaux } \\
\text { Bactérie hétérotrophe aérobie }\end{array}$ & & \\
\hline
\end{tabular}

des PE dans le BRM sont peu nombreuses (Tableau 8). Elles portent, pour la plupart, sur des composés phénoliques (ex. : nonylphénol, BPA), des hormones et des dérivés hormonaux (17 $\beta$-éthinylestradiol, œstradiol), quelques composés pharmaceutiques, etc. WINTGENS et al. (2002) ont rapporté, dans une étude d'évaluation du potentiel du BRM dans le traitement des eaux usées contenant des nonylphénols, que ces derniers sont réduits de $87 \%$. Une étude réalisée à l'échelle pilote par HU et al. (2007) indique que le bioréacteur membranaire élimine $80 \%$ à $90 \%$ d'oestrone (E1), $72 \%$ de $17 \alpha$-œstradiol (E2), et de $70 \%$ à $94 \%$ de BPA. Le taux d'abattement du BPA dans le BRM oscille généralement entre $93 \%$ et $97 \%$ (CHEN et al., 2008; CLARA et al., 2005a,b; LEE et al., 2008; LYKO et al., 2005).
LEE et al. (2008) indiquent que pendant que les nonylphénols, l'œstradiol et le $17 \beta$-éthinylestradiol sont éliminés à $55 \%, 64 \%$ et $\geq 71 \%$ respectivement, le BPA et la génistéine le sont à $93 \%$ et plus. Une étude effectuée à grande échelle par CLARA et al. (2005a) a confirmé ces résultats pour le $\mathrm{BPA}(\geq 93 \%)$ et le $17 \beta$-éthinylestradiol $(60 \%$ à $79 \%)$. Les produits pharmaceutiques et produits dérivés (médicaments, métabolites des médicaments, produits cosmétiques, compléments alimentaires et métabolites dérivés) ont également été mis en évidence, notamment le régulateur de lipides (benzafibrate), l'analgésique (ibuprofene), les composés polycycliques (galaxolide et tonalide) et l'antiépileptique (carbamazépine), pour ne citer que ceux-là (CLARA et al., 2004, 2005a). Les résultats de cette étude ont montré 
Tableau 8. Micropolluants organiques réfractaires dégradés par les BRM.

Table 8. Refractory organic micropollutants degraded by MBR.

\begin{tabular}{|c|c|c|c|}
\hline Matrices & Micropolluants & Enlèvement (\%) & Références \\
\hline EUS & Phénol & $100 \%$ & BARRIOS-MARTINEZ et al. (2006) \\
\hline EUS & Bisphénol A & $\geq 93,7 \%$ & CHEN et al. (2008) \\
\hline Lixiviat de décharge & Bisphénol A & $97 \%$ & LYKO et al. (2005) \\
\hline Lisier de porc & Composés oestrogéniques & $94,7 \%$ & YANG et CICEK (2008) \\
\hline Eau de surface & THM & $75 \%$ & LI et CHU (2003) \\
\hline EUM & E1 & $80 \%-91 \%$ & \multirow{7}{*}{ HU et al. (2007) } \\
\hline EUM & E2 & $63 \%$ & \\
\hline EUM & E1-3G & $62 \%-85 \%$ & \\
\hline EUM & E2-G & $55 \%-80 \%$ & \\
\hline EUM & E1-3S & $10 \%-48 \%$ & \\
\hline EUM & E2-3S & $80 \%-100 \%$ & \\
\hline EUM & $\mathrm{BPA}$ & $70 \%-94 \%$ & \\
\hline EUM & EE2 & $60 \%-79 \%$ & \multirow{3}{*}{ CLARA et al. (2004) } \\
\hline EUM & Benzafibrate, ibuprofene & $\geq 95 \%$ & \\
\hline EUM & Carbamazépine & $0 \%$ & \\
\hline EUM & Bisphénol A & $\geq 95 \%$ & CLARA et al. (2005a) \\
\hline Lixiviat de décharge & Nonylphénol & $87 \%$ & WINTGENS et al. (2002) \\
\hline EUM & Nonylphénol & $60 \%$ & \multirow{5}{*}{ LEE et al. (2008) } \\
\hline EUM & Génistéine & $97 \%$ & \\
\hline EUM & EE2 & $71 \%$ & \\
\hline EUM & E1 & $64 \%$ & \\
\hline EUM & Bisphénol A & $93 \%$ & \\
\hline EUM & EE2 & $80 \%-95 \%$ & \multirow{4}{*}{ ZUEHLKE et al. (2006) } \\
\hline EUM & Phénazone & $70 \%$ & \\
\hline EUM & Propyphénazone & $65 \%$ & \\
\hline EUM & Carbamazépine & $0 \%$ & \\
\hline
\end{tabular}

que benzafibrate et ibuprofène ont des taux d'élimination élevés ( $\geq 95 \%)$. Ensuite, suivent les composés polycycliques (tonalide et galaxolide), qui sont éliminés à près de $80 \%$. La carbamazépine n'est pas éliminée. Une étude à long terme effectuée par ZUEHLKE et al. (2006) a confirmé les résultats obtenus par CLARA et al. (2004, 2005a), en particulier pour le composé carbamazépine. Aussi, cette même étude rapporte que les composés tels que phénazone, propyphénazone et FAA sont partiellement éliminés dans le BRM (70 \%).

Les principales limites de la technologie seraient le dimensionnement, les exigences opératoires, les besoins accrus de maintenance et les coûts de fonctionnement. Les outils et modèles utilisés pour dimensionner les procédés à boues activées restent globalement applicables aux bioréacteurs à membranes (HENZE et al., 1987, 2000; HERBERT, 1958; METCALF et EDDY INC., 2003; PIRT, 1965). Cependant, le fait de travailler avec des $\theta$ et des concentrations en biomasse plus élevées oblige à réévaluer certains paramètres cinétiques pour intégrer : i) une accessibilité plus facile du substrat aux sites actifs due à une réduction des limitations au transfert induite par la présence d'organismes peu floculés; ii) une hydrolyse intensifiée des macromolécules (retenues par la membrane), et dont le temps de séjour est identique à celui de la fraction particulaire; et iii) une composition de la biomasse spécifique (GRASMICK et al., 2009).

Il paraît ainsi plus difficile de trouver un jeu de paramètres permettant de décrire la très large gamme de conditions opératoires offertes par les BRM. Aussi, les coûts d'investissement (coûts des membranes) et le colmatage ont longtemps limité l'application à grande échelle des BRM. Les progrès remarquables effectués au niveau de la technologie de fabrication des membranes font en sorte que les coûts d'investissement sont maintenant plus faibles. Toutefois, les coûts de fonctionnement demeurent encore élevés à cause du nettoyage fréquent et du remplacement des membranes, de l'aération très intensive appliquée pour le contrôle du colmatage, et de la consommation énergétique liée aux pompes de succion. Le BRM est également limité par la capacité de filtration des membranes. En effet, les débits d'eaux usées 
traitées par jour ne peuvent pas excéder les débits de filtration des membranes.

\subsection{Application à la réutilisation des eaux usées traitées}

Les BRM existent sous diverses formes dans les installations de traitement des eaux usées. Les performances sont en général très élevées et les eaux traitées sont de très bonne qualité, respectant les normes en vigueur en matière de réutilisation des eaux usées dans plusieurs pays du monde (États-Unis, Canada, France, etc.). Les effluents de BRM pourraient donc être réutilisés ou recyclés, dépendamment des usages spécifiques visés. Il existe déjà dans le monde plusieurs cas de réutilisation des effluents de BRM, aussi bien pour l'agriculture, l'irrigation des espaces verts que pour les eaux de toilettes, etc. La société Novidon (Veurne, Belgique), qui traite de l'amidon, utilise la technologie de BRM depuis 2003 pour le traitement de ses eaux usées. Ces eaux traitées (perméat) sont utilisées à plus ou moins $50 \%$, pour différentes applications dans la production (eau de rinçage, eau de lavage, etc.). Depuis 2008, le Pennant Hills Golf Club australien (Beecroft, New South Wales, Australie) réutilise à $98 \%$ les EUM pour l'irrigation des parcours de golf et des espaces verts. Ces EUM réutilisées sont des effluents d'un BRM immergé à boucle externe (ZeeWeed, Zenon). Elles respectent les normes australiennes en matière de réutilisation des eaux usées. Le Vancouver Convention and Exhibition Center (Vancouver, Canada) réutilise $100 \%$ des eaux usées produites dans son édifice (lesquelles sont traitées dans un BRM), pour l'irrigation des espaces verts. Depuis 1993, un projet de réutilisation des effluents de la STEP municipale de Santa Cruz (Tenerife Island), pour l'irrigation des plantations de bananes et de tomates, a été entrepris afin de rendre économiquement compétitives les cultures de cette région et celles de l'Amérique du Sud. L'intégration d'un système de BRM à la station de boues activées existante a permis d'obtenir des eaux qui respectent les garanties de santé acceptables par le marché international (VERA et al., 1997). La réutilisation des effluents de BRM peut aussi requérir des étapes supplémentaires de traitement afin d'obtenir une qualité respectant les usages spécifiques. Dans une telle situation, le BRM s'avère alors un bon prétraitement avant une étape d'osmose inverse. La réutilisation, au niveau de l'industrie agroalimentaire, reste encore un sujet tabou du fait des impératifs sanitaires de production.

\section{APPLICATIONS DU BRM À GRANDE ÉCHELLE}

Le tableau 9 présente quelques installations de BRM à travers le monde. Les BRM peuvent constituer le cœur $\mathrm{du}$ traitement, ou encore, servir d'étape d'affinage. La commercialisation du BRM immergé a accéléré la pénétration et l'extension des BRM sur le marché du traitement des eaux. En 2005, le BRM immergé représentait plus de $97 \%$ des installations utilisant les biotraitements membranaires en Europe. Le marché des BRM est en pleine croissance et avait, en 2005, une valeur de 217 millions de dollars américains, avec une croissance annuelle de plus de $10 \%$, plus rapide que les autres procédés avancés de traitement des eaux et autres types de systèmes membranaires (JUDD, 2008; LESJEAN et HUISJES, 2008). Dans le monde, on dénombre plus de 2200 systèmes de BRM destinés au traitement des eaux. Actuellement, le plus grand BRM est installé à la station d'épuration des EUM de Nordkanal à Kaarst, en Allemagne (débit maximum de $48000 \mathrm{~m}^{3} \bullet \mathrm{jr}^{-1}$ ) (LESJEAN et HUISJES, 2008; MELIN et al., 2006). En Amérique du Nord, il existait déjà plus de 15 stations d'épuration des eaux usées utilisant la technologie de BRM, avec des débits journaliers allant jusqu’à $9500 \mathrm{~m}^{3} \bullet \mathrm{jr}^{-1}$ (YANG et al., 2006). En Europe, il existait environ 100 stations de traitement des eaux d'une capacité supérieure à $500 \mathrm{EH}$ (équivalent-habitants), utilisant la technologie de BRM pour le traitement des EUM, et 300 autres pour le traitement des EUI, d'une capacité supérieure à $20 \mathrm{~m}^{3} \bullet \mathrm{jr}^{-1}$. En Europe, le débit moyen journalier traité est de $13000 \mathrm{~m}^{3} \bullet \mathrm{jr}^{-1}$ pour les STEP municipales utilisant des BRM, et de $2500 \mathrm{~m}^{3} \bullet \mathrm{jr}^{-1}$ pour les STEP industrielles. En Asie, en particulier au Japon et en Corée du Sud, les BRM sont majoritairement utilisés pour des applications à petite échelle (traitement de EUD). Le BRM devient de plus en plus abordable sur le plan économique, grâce à la diminution continue du coût de fabrication des membranes et des normes de rejets qui évoluent vers une sévérité croissante, aussi bien en ce qui concerne les concentrations en polluants que les débits. Il est estimé que le marché actuel doublera tous les sept ans. Il atteindra une valeur de 360 millions de dollars américains en 2010 .

\subsection{Systèmes commerciaux}

Plusieurs systèmes de BRM sont commercialisés dans le monde (Tableau 10). La pénétration et l'extension des bioréacteurs à membrane sur le marché du traitement des eaux sont en majorité dues à la commercialisation du BRM immergé. Les systèmes commercialisés sont, entre autres :

- le systèmeClereflo MBR(Conder Products, Royaume-Uni), pouvant être utilisé pour le traitement des eaux usées d'une municipalité ou d'une collectivité de $5000 \mathrm{EH}$;

- le système PURON ${ }^{\circledR}$ (Submerged Hollow Fiber Membrane Filtration, Koch membrane systems);

- les systèmes ZeeMOD et ZeeWeed, commercialisés par la 
Tableau 9. Exemples d'application à grande échelle des BRM dans le monde.

Table 9. BRM installations for wastewater treatment in the world.

\begin{tabular}{|c|c|c|c|c|c|}
\hline Localisations & Matrices & Caractéristiques & $\begin{array}{l}\text { Capacité } \\
\left(\mathrm{m}^{3} \bullet \mathrm{jr}^{-1}\right)\end{array}$ & $\begin{array}{l}\text { Date de mise } \\
\text { en service }\end{array}$ & Références \\
\hline $\begin{array}{l}\text { Kraarst, } \\
\text { Allemagne }\end{array}$ & $\begin{array}{l}\text { Eaux usées } \\
\text { municipales }\end{array}$ & Zenon, membrane en fibres creuses & 48000 & 2003 & LESJEAN et HUISJES (2008) \\
\hline $\begin{array}{l}\text { Traverse City, } \\
\text { États-Unis }\end{array}$ & $\begin{array}{l}\text { Eaux usées } \\
\text { municipales }\end{array}$ & Zenon, membrane en fibres creuses & 26900 & 2004 & YANG et al. (2006) \\
\hline $\begin{array}{l}\text { Johns Creek, } \\
\text { États-Unis }\end{array}$ & $\begin{array}{l}\text { Eaux usées } \\
\text { municipales }\end{array}$ & $\begin{array}{l}\text { Zenon, membrane en fibres creuses, } \\
\mathrm{p}=0,04 \mu \mathrm{m}, \mathrm{a}=31,6 \mathrm{~m}^{2}\end{array}$ & 40000 & & MELIN et al. (2006) \\
\hline Guilvinec, France & $\begin{array}{l}\text { Eaux usées } \\
\text { municipales }\end{array}$ & $\begin{array}{l}\text { Kubota, membrane en feuilles plates, } \\
\text { polyéthylène, pore }=0,4 \mu \mathrm{m}\end{array}$ & 2600 & 2004 & MELIN et al. (2006) \\
\hline $\begin{array}{l}\text { Varsseveld, NL, } \\
\text { États-Unis }\end{array}$ & $\begin{array}{l}\text { Eaux usées } \\
\text { municipales }\end{array}$ & Zenon, membrane en fibres creuses & 5000 & 2004 & MELIN et al. (2006) \\
\hline $\begin{array}{l}\text { Brescia, } \\
\text { Italie }\end{array}$ & $\begin{array}{l}\text { Eaux usées } \\
\text { municipales }\end{array}$ & Zenon, membrane en fibres creuses & 42000 & 2002 & MELIN et al. (2006) \\
\hline $\begin{array}{l}\text { Park Place, GA, } \\
\text { États-Unis }\end{array}$ & $\begin{array}{l}\text { Eaux usées } \\
\text { municipales }\end{array}$ & US Filter, HF, PVDF, pore $=0,1 \mu \mathrm{m}$ & 1000 & 2003 & YANG et al. (2006) \\
\hline Vaujany, France & $\begin{array}{l}\text { Eaux de captages et } \\
\text { eaux de surfaces }\end{array}$ & $\begin{array}{l}\text { Membrane en polypropylène, } \\
\text { pore }=0,2 \mu \mathrm{m}\end{array}$ & 4500 & 2000 & BERLAND et al. (2002) \\
\hline Porlock, UK & Eaux de vidanges & $\begin{array}{l}\text { Kubota, polymérique, support en } \\
\text { fibres, pore }=0,4 \mu \mathrm{m}\end{array}$ & 1900 & 1998 & GANDER et al. (2000) \\
\hline
\end{tabular}

Tableau 10. Liste non exhaustive des principaux fournisseurs de BRM (ORANTES et al., 2006; TAO et al., 2005).

Table 10. Non exhaustive list of BRM suppliers in the world (TAO et al., 2005; ORANTES et al., 2006).

\begin{tabular}{lll}
\hline Membranes plaques & Fibres creuses & Boucle externe \\
\hline A3 & Asahi Kasei & Multitube/multichannel \\
Brightwater & Ecologix & $\bullet$ \\
Huber & GE Zenon & $\bullet$ \\
KOReD & Koch Membrane System (Puron) & $\bullet$ \\
Kubota & Memcor & Orelis \\
Martin & Mitsubishi Rayon & Fibres creuses \\
Microdyn-Nadir & Polymem & Ultraflo \\
SINAP & Porous Fibers S.L & Disque céramique \\
Toray & Siemens & $\bullet$ \\
Vina Filter & Sumitomo & KERAFOL \\
Weise & Tianjin Motimo & Grundfos \\
\end{tabular}

compagnie Zenon-GE, et pouvant traiter des débits allant jusqu'à $7500 \mathrm{~m}^{3} \bullet \mathrm{jr}^{-1}$ pour le premier et $48000 \mathrm{~m}^{3} \bullet \mathrm{jr}^{-1}$ pour le second;

- les systèmes EFLO MBR (EFLO International Ltd, Royaume-Uni), qui sont des BRM immergés à membranes planes en polyéthersulfone, d'un diamètre de pores de $0,2 \mu \mathrm{m}$. Ces systèmes peuvent être installés dans ou sur le sol, ou peuvent être mobiles pour des utilisations temporaires. Ils permettent de traiter des débits allant de 120 à $1200 \mathrm{~m}^{3} \bullet \mathrm{jr}^{-1}$ selon les modèles.

- les systèmes BIO-CEL (Microdyn-Nadir, Wiesbaden, Allemagne), BRM immergés à membranes planes (PES, $0,04 \mu \mathrm{m})$;
- les systèmes AirLiftTM Membrane BioReactor (MBR) à boucle externe et Crossflow sont des BRM à membranes tubulaires, fonctionnant à débits élevés. Le système AirLiftTM Membrane BioReactor (MBR) est destiné au traitement des EUM, tandis que le système Crossflow est destiné au traitement des EUI et des lixiviats de décharge;

- le système Membrane-Biology Municipal Wastewater de Stulz-Planaqua (Allemagne) est un BRM immergé d'une capacité de $700 \mathrm{EH}$ et d'un débit maximal de $290 \mathrm{~m}^{3} \bullet \mathrm{jr}^{-1}$. Il est destiné au traitement des EUM;

le système MembrexTM (TECHNOLOGIES PREMIER TECH et HUBER TECHNOLOGY) est un BRM à membrane immergé équipé de membranes planes. 
Le marché des BRM est dominé par les fournisseurs de membranes suivants :

- Membranes planes : Kubota, A3, Toray, Martin systems, Microdyn-Nadir, Huber.

- Membranes à fibres creuses : KMS-Puron, Zenon-GE, Mitsubishi, Memcor, Asahi et US Filter.

- Membranes à fibres creuses sous pression : Rhodia-Orelis, Norit X et Wehrle environmental.

En Amérique du Nord, il y a quatre principaux fabricants de BRM. Ce sont : Zenon Environmental Inc. (Canada), USFilter (USA), Kubota (Japon) et Mitsubishi-Rayon (Japon). En Europe, les grands groupes présentent des systèmes clé en main : le Biosep (Veolia/Membrane Puron), l'Aqua-RM et le Compact-RM de STEREAU et le procédé Ultrafor de Degrémont (membrane Zenon ou Toray).

\subsection{Domaine de fonctionnement}

La plage de fonctionnement des BRM (âge des boues et teneur en MES) s'est rapidement restreinte suite à des problèmes d'agitation et mélange, et de transfert de matière (oxygène). En effet, même si le BRM permet de dissocier TRH et TRS, un âge des boues élevé reste synonyme de teneur en MES élevées. De plus, de simples bilans matières montrent que des TRS importants concentrent la matière inerte (non utile pour la bioréaction) dans le réacteur (LOBOS et al., 2009). Ces composés s'accumulent suivant le facteur de concentration (TRS/TRH) et leurs concentrations initiales. La simulation numérique (Figure 4) montre que pour une charge donnée, la teneur en MES évolue presque linéairement avec le temps de rétention de solides. Cependant, la puissance d'aération, pour respecter la quantité d'air nécessaire à l'activité biologique, augmente de $132 \%$ quand on passe de $4 \mathrm{~g} \bullet \mathrm{L}^{-1}$ à $12 \mathrm{~g} \bullet \mathrm{L}^{-1}$. Cette surconsommation énergétique a ainsi contraint les exploitants de BRM à revoir leurs objectifs pour finalement adopter des TRS compris entre 20 et 30 jours, et donc des teneurs en MES de l'ordre de 6 à $8 \mathrm{~g} \bullet \mathrm{L}^{-1}$.

\subsection{Coûts d'installation et d'exploitation}

Les coûts de production, d'installation et d'exploitation d'une filière de traitement basée sur la technologie de BRM sont plus élevés comparativement aux autres systèmes de traitement conventionnel (ex. : système de boues activées) du fait, entre autres, de l'injection d'air (air-membrane) pour pérenniser l'étape de filtration. À l'heure actuelle, la production et l'installation d'une filière d'assainissement décentralisée, basée sur la technologie de BRM, se situe entre $3000 €$ et $97000 €$, dépendamment de la population desservie (6 à 200 personnes) (Tableau 11). Ces coûts dépendent de la taille du système et principalement des coûts des différents équipements mis en œuvre (pompes d'alimentation, modules membranaires, pompes de soutirage du perméat, surpresseurs d'air pour aération des membranes). Les frais annuels d'exploitation sont, quant à eux, liés à la consommation énergétique et la maintenance. Ils s'élèvent en moyenne à $1080 €$. Ils sont identiques à ceux d'un système traditionnel d'assainissement décentralisé. Le tableau 12 présente les coûts annuels liés à l'exploitation de différentes tailles de BRM. On remarque que les BRM immergés (utilisant des membranes en fibres creuses et membranes planes) reviennent moins chers en matière de coûts d'exploitation que les BRM à boucle externe (utilisant des membranes tubulaires). Cependant, les BRM à boucle externe sont caractérisés par leur simplicité d'exploitation et des coûts de production et d'installation plus faibles.
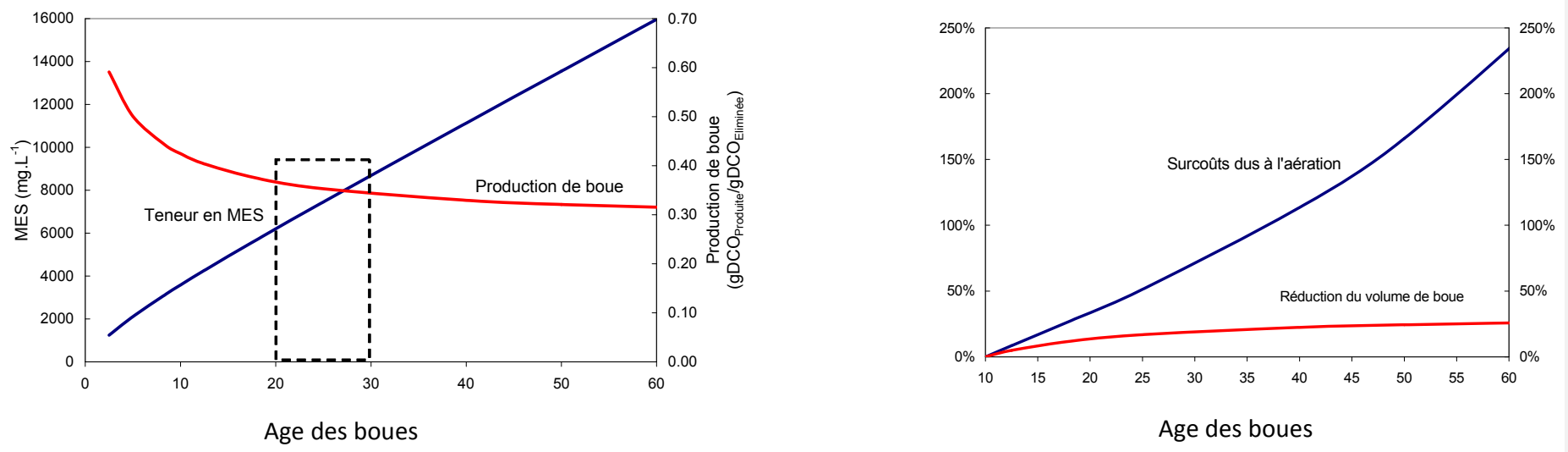

Figure 4. Influence des paramètres opératoires sur la conduite d'un bioréacteur à membrane (LOBOS et al., 2009. Influence of the operating parameters on the behaviour of a membrane bioreactor (LOBOS et al., 2009). 
Tableau 11. Coûts d'installation et consommation énergétique des BRM dans une filière d'assainissement décentralisé (adapté d'EUROMBRA, 2006).

Table 11. Equipment costs and power requirement of BRM for a decentralized wastewater treatment plant (adapted from EUROMBRA, 2006).

\begin{tabular}{|c|c|c|c|}
\hline $\begin{array}{c}\text { Capacité } \\
(\mathrm{EH})\end{array}$ & Types de systèmes & $\begin{array}{l}\text { Coûts énergétiques } \\
\text { (Euros) }\end{array}$ & $\begin{array}{c}\text { Coûts d'installation } \\
\text { (Euros) }\end{array}$ \\
\hline \multirow{3}{*}{6} & BRMi P & 139 & 4918 \\
\hline & BRMi FC & 143 & 4431 \\
\hline & BRMe T & 493 & 3870 \\
\hline \multirow{2}{*}{20} & BRMi FP & 463 & 11429 \\
\hline & BRMi FC & 477 & 8088 \\
\hline \multirow{3}{*}{50} & BRMi FP & 1737 & 27070 \\
\hline & BRMi FC & 1788 & 18947 \\
\hline & BRMe T & 6168 & 16811 \\
\hline \multirow{3}{*}{100} & BRMi FP & 3475 & 50904 \\
\hline & BRMi FC & 3578 & 34307 \\
\hline & BRMe T & 12337 & 29854 \\
\hline \multirow{3}{*}{200} & BRMi FP & 6951 & 97559 \\
\hline & BRMi FC & 7161 & 65625 \\
\hline & BRMe T & 24675 & 57332 \\
\hline
\end{tabular}

\begin{tabular}{ll}
\hline BRMi FC : & BRM immergé avec membranes à fibres creuses \\
BRMi P : & BRM immergé avec membranes planes \\
BRMe T : & BRM à membrane à boucle externe avec membranes tubulaires
\end{tabular}

Tableau 12. Coûts annuels par équivalent-habitant $(\mathrm{EH})$ pour trois types de modules membranaires dans une filière d'assainissement décentralisé (adapté d'EUROMBRA, 2006)

Table 12. Annual cost perperson-equivalent for three membrane systems in a decentralized wastewater treatment plant (adapted from EUROMBRA, 2006).

\begin{tabular}{|c|c|c|c|c|}
\hline Types de membranes & $\begin{array}{c}\text { Capacité } \\
(\mathrm{EH})\end{array}$ & $\begin{array}{c}\text { Coûts } \\
\left(\text { Euros }^{\bullet} \mathrm{EH}^{-1} \bullet \mathrm{an}^{-1}\right)\end{array}$ & $\begin{array}{c}\text { CAPEX } \\
\left(\text { Euros }^{-E^{-1}}\right)\end{array}$ & $\begin{array}{c}\mathrm{OPEX} \\
\left(\text { Euros }^{\bullet} \mathrm{EH}^{-1} \bullet \mathrm{an}^{-1}\right)\end{array}$ \\
\hline \multirow{5}{*}{ Feuilles plates } & 6 & 265 & 819 & 205 \\
\hline & 20 & 135 & 571 & 93 \\
\hline & 49 & 100 & 485 & 65 \\
\hline & 50 & 138 & 541 & 97 \\
\hline & 200 & 112 & 488 & 75 \\
\hline \multirow{5}{*}{ Fibres creuses } & 6 & 271 & 738 & 206 \\
\hline & 20 & 131 & 404 & 94 \\
\hline & 49 & 93 & 302 & 65 \\
\hline & 50 & 132 & 379 & 98 \\
\hline & 200 & 107 & 328 & 76 \\
\hline \multirow{5}{*}{ Tubulaires } & 6 & 304 & 645 & 246 \\
\hline & 20 & 170 & 347 & 134 \\
\hline & 49 & 131 & 241 & 105 \\
\hline & 50 & 193 & 304 & 158 \\
\hline & 200 & 167 & 257 & 136 \\
\hline
\end{tabular}

CAPEX : $\quad$ Capital expenditure ou dépenses d'investissement

OPEX : $\quad$ Operational expenditure ou dépenses de fonctionnement, d'exploitation

\section{CONCLUSION}

Le BRM est une technologie révolutionnaire dans le domaine du traitement des eaux. Il se caractérise par des performances intéressantes en matière d'élimination de polluants biodégradables et de polluants considérés comme difficilement biodégradables par les procédés usuels de traitement des eaux usées. Les eaux traitées par le BRM ont une excellente qualité en matière de MES et de désinfection. La technologie de BRM est relativement plus coûteuse que les systèmes traditionnels de boues activées, mais des efforts énormes ont été effectués (réduction significative du coût des membranes et des coûts d'exploitation) afin d'assurer son développement industriel et commercial. La plage de fonctionnement des BRM (âge des boues et teneur en MES) s'est rapidement restreinte suite à des problèmes d'agitation et de mélange, et de transfert de matière (oxygène). La surconsommation énergétique lors de 
l'opération des BRM a ainsi contraint les exploitants de BRM à revoir leurs objectifs pour finalement adopter des TRS compris entre 20 et 30 jours, et donc des teneurs en MES de l'ordre de 6 à $8 \mathrm{~g} \bullet \mathrm{L}^{-1}$. Toutefois, à ce jour, la technologie est principalement compétitive pour le traitement des EUI où les effluents sont très chargés. Pour les EUM, la demande énergétique élevée de $30 \%$ à $50 \%$ (en comparaison avec le système conventionnel de boues activées) peut s'avérer être un frein à l'utilisation de cette technologie. Ce frein se retrouve réduit devant la compacité d'une installation de BRM (réduction de l'emprise au sol d'un facteur quatre) et les performances du procédé (comparable à un traitement tertiaire). De plus, de nombreuses équipes de recherche travaillent pour développer et optimiser la technologie de BRM, afin de la rendre plus compétitive et adaptée aux applications municipales.

\section{RÉFÉRENCES BIBLIOGRAPHIQUES}

AMEDEUS (2008). Accelerated membrane development for urban sewage purification. D38: Final report WP6 Implementation of submerged module inside or outside of reactor. Proposal/Contract no.: 018328 AMEDEUS.

ANDREOZZI R., R. CESARO, R. MAROTTA et F. PIROZZI (2006). Evaluation of biodegradation kinetic constants for aromatic compounds by means of aerobic batch experiments. Chemosphere, 62, 1431-1436.

BAEK S.H. et K.R. PAGILLA (2008). Simultaneous nitrification and denitrification of municipal wastewater in aerobic membrane bioreactors. Water Environ. Res., 80, 109-117.

BARRIOS-MARTINEZ A., E. BARBOT, B. MARROT, P. MOULIN et N. ROCHE (2006). Degradation of synthetic phenol-containing wastewaters by BRM. J. Membr. Sci., 28, 288-296.

BATTISTONI P., F. FATONE, D. BOLZONELLA et P. PAVAN (2006). Full scale application of coupled alternate cycles-membrane bioreactor (AC-BRM) process for wastewater reclamation and reuse. Water Pract. Technol., IWA Publishing, doi10.2166/wpt.2006.0077.

BERLAND J.M. et C. JUERY (2002). Les procédés membranaires pour le traitement de l'eau. Document technique, FNDAE, $\mathrm{N}^{\circ}$ 14, Ministère de l'Agriculture, de l'Alimentation, de la Pêche et des Affaires rurales, Direction de l'Espace Rural et de la Forêt, France, 71 p.

BIRIMA A.H., M.J. MEGAT MOHD NOOR, A.S. MUYIBI et A. IDRIS (2005). Simultaneous organic and nitrogen removal using anoxic-aerobic membrane bioreactor. Int. J. Eng. Technol., 2, 36-42.

BREPOLS C., H. SCHÄFER et N. ENGELHARDT (2005). Hinweise zur verfahrenstechnischen Integration getauchter Membranfilter in kommunalen Membranbelebungsanlagen, $K A$ - Abwasser, Abfall, 52, 45-50.

BOUCHARD C., P. KOUADIO, D. ELLIS, M. RAHMI et R.E. LEBRUN (2000). Les procédés à membranes et leurs applications en production d'eau potable. Vecteur Environ., $33,28-38$.

BRINDLE K. et T. STEPHENSON (1996). The application of membrane biological reactors for the treatment of wastewaters. Biotechnol. Bioeng., 49, 601-610.

BUISSON H., T. LEBEAU, C. LELIEVRE et L. HRREMANS (1998). Les membranes: Point sur les évolutions d'un outil incontournable en production d'eau potable. Eau, Industrie, Nuisances, 210, 42-47.

CARBALLA M., F. OMIL et J.M. LEMA (2005). Removal of cosmetic ingredients and pharmaceuticals in sewage primary treatment. Water Res., 39, 4790-4796.

CARDOT C. (1999). Les traitements de l'eau : Procédés physico-chimiques et biologiques. Cours et problèmes résolus, ELLIPSES MARKETING (Éditeur), Technosup, Paris, France, $256 \mathrm{p}$.

CHANG H.-S., K.-H. CHOOA, B. LEEB et S.-J. CHOIA (2009). Review: The methods of identification, analysis, and removal of endocrine disrupting compounds (EDCs) in water. J. Hazard. Mater., 172, 1-12.

CHANG I.S. et S.N. KIM (2005). Wastewater treatment using membrane filtration effect of biosolids concentration on cake resistance. Process Biochem., 40, 1307-1314.

CHANG I.S., C.H. LEE et K.H. AHN (1999). Membrane filtration characteristics in membrane coupled activated sludge system: The effect of floc structure on membrane fouling. Sepac. Sci. Technol., 34, 15-30.

CHEE-SANFORD J.C., R.I. ARMINOV, I.J. KRAPAC, N. GARRIGUES-JEANJEAN et R.I. MACKIE (2001). Occurrence and diversity of tetracycline resistance genes in lagoon and groundwater underlying two swine production facilities. Appl. Environ. Microbiol., 67, 1494-1502.

CHEN J., X. HUANG et D. LEE (2008). Bisphenol A removal by a membrane bioreactor. Process Biochem., 43, 451-456. 
CHO B.D. et A.G. FANE (2002). Fouling transients in nominally sub-critical flux operation of a membrane bioreactor. J. Membr. Sci., 209, 391-403.

CHOI H., D.D. DIONYSIOS, D.B. OERTHER et G.A. SORIAL (2005). Influence of cross flow velocity on membrane performance during filtration of biological suspension. J. Membr. Sci., 248, 189-199.

CHOO K.H. et C.H. LEE (1996). Membrane fouling mechanisms in the membrane-coupled anaerobic bioreactor. Water Res., 30, 1771-1780.

CICEK N., H. WINNEN, M.T. SUIDAN, B.E. WRENN, V. URBAIN et J. MANEM (1998). Effectiveness of the membrane bioreactor in the degradation of high molecular weight compounds. Water Res., 32, 1553-1563.

CIRJA M., P. IVASHECHKIN, A. SHAFFER et P.F.X. CORVINI (2008). Factors affecting the removal of organic micropollutants from wastewater in conventional treatment plants (CTP) and membrane bioreactors (BRM). Rev. Environ. Sci. Biotechnol., 7, 61-78.

CLARA M., B. STRENN, M. AUSSERLEITNER et N. KREUZINGER (2004). Comparison of the behaviour of selected micropollutants in a membrane bioreactor and a conventional wastewater treatment plant. Water Sci. Technol., 50, 29-36.

CLARA M., B. STRENN, O. GANS, E. MARTINEZ, N. KREUZINGER et H. KROISS (2005a). Removal of selected pharmaceuticals, frangrances and endocrine disrupting compounds in membrane bioreactor and conventional wastewater treatment plant. Water Res., 39, 4797-4807.

CLARA M., N. KREUZINGER, B. STRENN, O. GANS et H. KROISS (2005b). The solids retention time - a suitable design parameter to evaluate the capacity of wastewater treatment plant to remove micropollutants. Water Res., 39, 97-106.

COMEAU Y. (2006). Traitement tertiaire (polissage) du lisier de porc par un bioréacteur à membranes(BRM) immergées. Rapport N ${ }^{\circ}$ 703035, École Polytechnique de Montréal, Montréal, QC, Canada, 142 p.

COTE P., H. BUISSON, C. POUND et G. ARAKAKI (1997). Immersed membrane activated sludge for the reuse of municipal wastewater. Desalination, 113, 189-196.
DEFRANCE L. et M.Y. JAFFRIN (1999). Reversibility of fouling formed in activated sludge filtration. J. Membr. Sci., 157, 73-84.

DEFRANCE L., M.Y. JAFFRIN, B. GUPTA, P. PAULLIER et V. GEAUGEY (2000). Contribution of various constituents of activated sludge to membrane bioreactor fouling. Bioresour. Technol., 73, 105-112.

DELGADO S., F. DIAZ, R. VILLARROEL, L. VERA, R. DIAZ et S. ELMALEH (2002). Nitrification in a hollow-fibre membrane bioreactor. Desalination, 146, 445-449.

DHAOUADI $\mathrm{H}$. et B. MARROT (2008). Olive mill wastewater treatment in a membrane bioreactor: Process feasibility and performances. Chem. Eng. J., 145, 225-231.

ESPLUGAS S., D.M. BILA, L.G.T. KRAUSE etM.DEZOTTI (2007). Ozonation and advanced oxidation technologies to remove endocrine disrupting chemicals (EDCs) and pharmaceuticals and personal care products (PPCPs) in water effluents. J. Hazard. Mater., 149, 631-642.

EUROMBRA (2006). Membrane bioreactor technology (MBR) with an EU perspective for advanced municipal wastewater treatment strategies for the $21^{\text {st }}$ century. D16 - Cost analysis, literature data (incl. pilot plant trials conducted by partners). Projet No 018480, European Commission.

FANEA.G., C.J.D. FELLet M.T.NOR(1980). Ultrafiltration/ activated sludge system - development of a predicted model. Dans : Ultrafiltration Membranes and Applications. COOPER A.R. (Éditeur), Plenum Press, New York, NY, États-Unis, pp. 631-648.

GANDER M., B. JEFFERSON et S. JUDD (2000). Aerobic MBRs for domestic wastewater treatment: a review with cost considerations. Sepac. Purif. Technol., 18, 119-130.

GARCIA M.T., E. CAMPOS, M. DALMAUl, I. RIBOSA et J.SANCHEZ-LEAL (2002). Structure activity relationships for association of linear alkylbenzene sulfonates with activated sludge. Chemosphere, 49, 279-286.

GERECKE A.C., M. SCHARER, H.P. SINGER, S.R. MULLER, R.P. SCHWARZENBACH, M. SAGESSER, U. OCHSENBEIN et G. POPOW (2002). Sources of pesticides in surface waters in Switzerland: Pesticide load through wastewater treatment plants current situation and reduction potential. Chemosphere, 48, 307-315. 
GIGER W., A.C. ALDER, E.M. GOLET, H.P.E. KOHLER, C.S. McARDELL, E. MOLNAR, H. SIEGRIST et M.J.F. SUTER (2003). Occurrence and fate of antibiotics as trace contaminants in wastewaters, sewage sludges and surface waters. Chimia, 59, 485-491.

GOGATE P.R. et A.B. PANDIT (2004). A review of imperative technologies for wastewater treatment I: oxidation technologies at ambient conditions. $A d v$. Environ. Res., 8, 501-551.

GRASMICK A., C. CABASSUD, M. SPÉRANDIO et C. WISNIEWSKI (2009). Les bioréacteurs à membrane appliqués au traitement des eaux usées. Les techniques de l'ingénieur, Réf. W4140, Service Relations Clients, Éditions Techniques de l'ingénieur, Paris, France.

HENZE M., P.L. GRADYC, W. GUJER, G.V.R. MARAIS et T. MATSUO (1987). Activated sludge model $N^{\circ} 1$. IAWPRC Scientific and Technical Reports $N^{\circ} 1$, Londres, Royaume-Uni.

HERBERT D. (1958). Some principles of continuous culture. Dans : Recent Progress in Microbiology. $7^{\text {th }}$ International Congress on Microbiology, G. TUNEVALL (Éditeur), Almquist et Wiksell, Stockholm, Suède, pp. 381-396.

HOLAKOO L., G. NAKHLA, A.S. BASSI et E.K. YANFUL (2007). Long term performance of BRM for biological nitrogen removal from synthetic municipal wastewater. Chemosphere, 66, 849-857.

HU J.Y, X. CHEN, G. TAO et K. KEKRED (2007). Fate of endocrine disrupting compounds in membrane bioreactor systems. Environ. Sci. Technol., 41, 4097-4102.

IKEHATA K et M.G. EL-DIN (2006) Aqueous pesticide degradation by hydrogen peroxide/ultraviolet irradiation and Fenton-type advanced oxidation processes: a review. J. Environ. Eng. Sci., 5, 81-135.

ILANI T., E. SCHULZ et B. CHEFETZ (2005). Interactions of organic compounds with wastewater dissolved organic matter: role of hydrophobic fractions. Environ. Qual., 34, 552-562.

JAWAD H.A.R. (2008). Performance of water recycling technology. Thèse de doctorat, University of Wollongong, No 43434677, New South Wales, Australie, 172 p.

JUDD S. (2008). The status of membrane bioreactor technology. Trends Biotechnol., 26, 109-116.
KAAM R.V., D. ANNE-ARCHARD, M. ALLIET, S. LOPEZ et C. ALBASI (2006). Aeration mode, shear stress and sludge rheology in a submerged membrane bioreactor: some keys of energy saving. Desalination, 199, 482-484.

LEBEGUE L., M. HERAN, A. GRASMICK (2009). Membrane air flow rates and HF sludging phenomenon in SMBR. Desalination, 236, 135-142.

LE-CLECH P., V. CHEN et A.G. FANE TONY (2006). Fouling in membrane bioreactors used in wastewater treatment (review). J. Membr. Sci., 28, 17-53.

LEE J., B.C. LEE, J.S. RA, J. CHO, I.S. KIM, N.I. CHANG, H.K. KIM et S.D. KIM (2008). Comparison of the removal efficiency of endocrine disrupting compounds in pilot scale sewage treatment processes. Chemosphere, 71, 1582-1592.

LEE W., S. KANG et H. SHIN (2003). Sludge characteristics and their contribution to microfiltration in submerged membrane bioreactors. J. Membr. Sci., 216, 217-227.

LEE Y., J. CHO, Y. SEO, J.W. LEE et K.H. AHN (2002). Modelling of submerged membrane bioreactor process for wastewater treatment. Desalination, 146, 451-457.

LESAGE N., M. SPÉRANDIO et C. CABASSUD (2005). Performances of a hybrid adsorption/submerged membrane biological process for toxic waste removal. Water Sci. Technol., 51, 173-180.

LESJEAN B. et E.H. HUISJES (2008). Survey of the European BRM market: trends and perspectives. Desalination, 231, 71-81.

LESJEAN B., V. FERRE, E. VONGHIA et H. MÖSLANG (2008). Market and design considerations of the 37 larger MBR plants in Europe. Dans: EDS MDIW08 Conference, 20-22 octobre, Toulouse, France.

LI X., F. GAO, Z. HUA, G. DU et J. CHEN (2005). Treatment of synthetic wastewater by a novel MBR with granular sludge developed for controlling membrane fouling. Sepac. Purif. Technol., 46, 19-25.

LI X.Y. et H.P. CHU (2003). Membrane bioreactor for the drinking water treatment of polluted surface water supplies. Water Res., 37, 4781-4791.

LI X.Y. et X.M. WANG (2006). Modelling of membrane fouling in a submerged membrane bioreactor. J. Membr. Sci., 278, 151-161. 
LIANG S., L. SONG, T. TAO, K.A. KEKRE et H. SEAH (2006). A modeling study of fouling development in membrane bioreactors for wastewater treatment. Water Environ. Res., 78, 853-863.

LINDBERG R.H., U. OLOFSSON, P. RANDAHL, M. JOHANSSON, M. TYAKLIND et B.A.V. ANDERSSON (2006). Behaviour of fluoroquinolones and trimethoprim during mechanical, chemical and activated sludge treatment of sewage and digestion of sludge. Environ. Sci. Technol., 40,1042-1048.

LIU R., X. HUANG, Y.F. SUN et Y. QIAN (2003). Hydrodynamic effect on sludge accumulation over membrane surfaces in a submerged membrane bioreactor. Proc. Biochem., 39(2), 157-163.

LIU Z. H, Y. KANJO et S. MIZUTANI (2009). Removal mechanisms for endocrine disrupting compounds (EDCs) in wastewater treatment-physical means, biodegradation, and chemical advanced oxidation: A review. Sci. Total Environ., 40, 731-748.

LOBOS J., M. HERAN et A. GRASMICK (2009), Optimization of the operations conditions in membrane bioreactors through the use of ASM3 model simulations. Desalin. Water Treat., 9, 126-130.

LYKO S., T. WINTGENS et T. MELIN (2005). Estrogenic trace contaminants in wastewater - Possibilities of membrane bioreactor technology. Desalination, 178, 95-105.

LYMAN W.J. (1990). Handbook of chemical property estimation methods: Environmental behavior of organic compounds. American Chemical Society, Washington, DC, États-Unis, pp. 1.1-1.54.

MARROT B., A. BARRIOS-MARTINEZ, P. MOULIN et N. ROCHE (2004). Industrial wastewater treatment in a membrane bioreactor: A review. Environ. Prog., 23, 59-68.

MATOSIC M., M. VUKOVIC, M. CURLIN et I. MIJATOVIC (2008). Fouling of hollow fiber submerged membrane during a long-term filtration activated sludge. Desalination, 219, 57-65.

MCBRIEN M.A., E. KOLOVANOV et V. TASHLTSSKY (2004). Application of structure - based pKa perdiction to reverse phase chromatigraphic method development. Ad. Chem. Dev., http://www.acdlabs.com/download/ publ/2004/cpsa04_pka.pdf.
MELIN T., B. JEFFERSON, D. BIXIO, C. THOEYE,

W. DE WILDE, J. DE KONING, J. VAN DER GRAAF et T. WINTGENS (2006). Membrane bioreactor technology for wastewater treatment and reuse. Desalination, 187, 271-282.

MENG F., H. ZHANG, Y. LI, X. ZHANG et F. YANG (2005). Application of fractal permeation model to investigate membrane fouling in membrane bioreactor. J. Membr. Sci., 262, 107-116.

METCALF et EDDY INC. (2003). Wastewater Engineering. McGRAW HILL, New York, NY, États-Unis, 1819 p.

MOULIN C. (1990). Potabilisation d'une eau de surface par filtration tangentielle sur membrane minérale : étude de traitements physico-chimiques associés. Thèse de doctorat, Université Montpellier, Montpellier, France, 180 p.

NAGANO A., E. ARIKAWA et H. KOBAYASHI (1992). The treatment of liquor wastewater containing high strength suspended solids by membrane bioreactor system. Water Sci. Technol., 26, 887-895.

NG A.N.L. et A.S. KIM (2007). A mini-review of modeling studies on membrane bioreactor (MBR) treatment for municipal wastewaters. Desalination, 212, 261-281.

OGNIER S., C. WISNIEWSKI et A. GRASMICK (2004). Membrane bioreactor fouling in sub-critical filtration conditions: a local critical flux concept. J. Membr. Sci., 229, 171-177.

ORANTES J., C. WISNIEWSKI, M. HERAN et A. GRASMICK (2006). The influence of operating conditions on permeability changes in a submerged membrane bioreactor. Sepac. Purif. Technol., 52, 60-66.

PARSONS S. (2004). Advanced oxidation processes for water and wastewater treatment. IWA Publishing, Alliance House, Londres, Angleterre, 356 p.

PELLEGRIN M.L., C. WISNIEWSKI, A. GRASMICK, A. TAZI-PAIN et H. BUISSON (2002). Sequenced aeration in a membrane bioreactor: specific nitrogen removal rates. Can. J. Chem. Eng., 80, 386-392.

PIRT S.J. (1965). The maintenance energy of bacteria in growing cultures. Proc. Royal Soc. London, 163(B), 224-231.

POCHANNA K., J. KELLER et P. LANT (1999). Model development for simultaneous nitrification and denitrification. Water Sci. Technol., 39, 235-243. 
POUET M.F., F. PERSIN et M. RUMEAU (1992). Intensive treatment by electrocoagulation-flottation-tangential flow microfiltration areas of high seanoal population. Water Sci. Technol., 25, 247-253.

RACZ L.A. et R.K. GOEL (2010). Fate and removal of estrogens in municipal wastewater. J. Environ. Monit., 12, 58-70.

RAMIREZ J.A. et R.H. DAVIS (1998). Application of crossflow microfiltration with rapid backpulsing to wastewater treatment. J. Hazard. Mater., B(36), 179-197.

REEMTSMA T., B. ZYWICKI, M. STUEBER, A. KLOEPFER et M. EKEL (2002). Removal of sulphurorganic polar micropollutants in a membrane bioreactor treating industrial wastewater. Environ. Sci. Technol., 36, 1102-1106.

SNYDER S. A., P. WESTERHOFF, Y. YOON et D. SEDLAK (2003). Pharmaceuticals, personal care products, and endocrine disruptors in water: implications for the water industry. Environ. Eng. Sci., 20, 449-469.

SORENSEN H.B. et E.S. JORGENSEN (1993). The removal of nitrogen compounds from wastewater. ELSEVIER SCIENCE (Éditeur) B.V., Amsterdam, Pays-Bas, 119 p.

STEPHENSON T., S. J JUD, B. JEFFERSON et K. BRINDLE (2000). Membrane bioreactors for wastewater treatment. IWA (Éditeur), Londres, Royaume-Uni, 192 p.

TAO G., K. KEKRE, Z. WEI, T.C. LEE, B. VISWANATH et H. SEAH (2005). Membrane bioreactors for water reclamation. Water Sci. Technol., 51, 431-440.

TARDIEU E., A. GRASMICK, V. GEAUGEY et J. MANEM (1999). Influence of hydrodynamics on fouling velocity in a recirculated MBR for wastewater treatment. J. Membr. Sci., 156, 131-140.

TIXIER C., H.P. SINGER, S. OLLERS et S.R. MULLER (2003). Occurrence and fate of carbamazepine, clofibric acid, diclofenac, ibuprofen, ketoprofen, and naproxen in surface waters. Environ. Sci. Technol., 37, 1061-1068.

VAN BENTEM A.G.N., C.P. PETRI, P.F.T. SCHYNS et H. F. VAN DER ROEST (2007). Membrane bioreactors. Operation and results of an MBR wastewater treatment plant. STOWA report, IWA (Éditeur), London, UK, 108 p.
VERA L., R. VILLAROEL-LOPEZ, S. DELGADO et S. ELMALEH (1997). Cross-flow microfiltration of biologically treated wastewater. Desalination, 114, 65-75.

VERA L., R. VILLARROEL, S. DELGADO et S. ELMALEH (2000). Enhancing microfiltration through an inorganic tubular membrane by gaz sparging. J. Membr. Sci., 165, $47-57$.

WINTGENS T., J. ROSEN, T. MELIN, C. BREPOLS, K. DRENSLA et N.ENGELHARDT (2003). Modelling of a membrane bioreactor system for municipal wastewater treatment. J. Membr. Sci., 216, 55-65.

WINTGENS T., M.GALLENKEMPER et T. MELIN (2002). Endocrine disrupter removal from wastewater using membrane bioreactor and nanofiltration technology. Desalination, 146, 387-391.

XING C.H., E. TARDIEU, Y. QIAN et X.H.WEN (2000). Ultrafiltration membrane bioreactor for urban wastewater reclamation. J. Membr. Sci., 177, 73-82.

YAMAMOTO K., M. HIASA, T. MAHMOOD et T. MATSUO (1989). Direct solid-liquid separation using hollow fiber membrane in an activated sludge aeration tank. Water Sci. Technol., 21, 43-54.

YANG W. et N. CICEK (2008). Treatement of swine water by submerged membrane bioreactors with consideration of estrogenic activity removal. Desalination, 231, 200-208.

YANG W., N. CICEK et J. ILG (2006). State-of-the-art of membrane bioreactors: Worldwide research and commercial applications in North America. J. Membr. Sci., 270, 201-211.

YU Z. et W. HUANG (2005). Competitive sorption between 17-alpha-ethinyl estradiol and naphthalene/phenanthrene by sediments. Environ. Sci. Technol., 39, 4878-4885.

YU K., X. WEN, Q. BU et H. XIA (2003). Critical flux enhancements with air sparging in axial hollow fibers cross-flow microfiltration of biological treated wastewater. J. Membr. Sci., 224, 69-79.

ZAVISKA F., P. DROGUI, G. MERCIER, et J.-F. BLAIS (2009). Procédé d'oxydation avancée dans le traitement des eaux et des effluents industriels : Application à la dégradation des polluant réfractaires. Rev. Sci. Eau, 22, 535-564.

ZHANG D. et W. VERSTRAETE (2002). The treatment of high strength wastewater containing high concentrations 
of ammonium in a staged anaerobic and aerobic membrane bioreactor. J. Environ. Eng. Sci., 1, 303-310.

ZHANG S., F. YANG, Y. LIU, X. ZHANG, Y. YAMADA et K. FURUKAWA (2006). Performance of a metallic membrane bioreactor treating simulated distillery wastewater at temperatures of 30 to $45^{\circ} \mathrm{C}$. Desalination, 194, 146-155.

ZUEHLKE S., U. DUENNBIER, R. LESJEAN et H. BUISSON (2006). Long-term comparison of trace organics removal performances between conventional and membrane activated sludge processes. Water Environ. Res., 78, 2480-2486. 